Delft University of Technology

\title{
Convex Optimization Guidance for Precision Landing on Titan
}

Mazouz, Rayan; Quadrelli, Marco B.; Mooij, E.

DOI

10.2514/6.2021-1345

Publication date

2021

Document Version

Final published version

Published in

AIAA Scitech 2021 Forum

\section{Citation (APA)}

Mazouz, R., Quadrelli, M. B., \& Mooij, E. (2021). Convex Optimization Guidance for Precision Landing on Titan. In AIAA Scitech 2021 Forum: 11-15 \& 19-21 January 2021 Virtual/online event [AIAA 2021-1345] American Institute of Aeronautics and Astronautics Inc. (AIAA). https://doi.org/10.2514/6.2021-1345

\section{Important note}

To cite this publication, please use the final published version (if applicable).

Please check the document version above.

\section{Copyright}

Other than for strictly personal use, it is not permitted to download, forward or distribute the text or part of it, without the consent of the author(s) and/or copyright holder(s), unless the work is under an open content license such as Creative Commons.

\section{Takedown policy}

Please contact us and provide details if you believe this document breaches copyrights.

We will remove access to the work immediately and investigate your claim. 


\title{
Convex Optimization Guidance for Precision Landing on Titan
}

\author{
Rayan Mazouz* and Marco B. Quadrelli ${ }^{\dagger}$ \\ NASA Jet Propulsion Laboratory, California Institute of Technology, Pasadena, California 91109 \\ Erwin Mooij \\ Delft University of Technology, Delft, The Netherlands
}

Precision landing is an anticipated technology for future interplanetary missions. Autonomous spacecraft Entry, Descent and Landing (EDL) on the surface of a planetary body with a degree of precision in the order of meters is highly challenging. In this paper, a successive convexification guidance algorithm is utilized to simulate autonomous precision landing sequences on Saturn's moon Titan. Due to its unique geophysical features, studying the science of matter within Titan's atmosphere and beneath its surface is one of NASA's most important planetary science objectives. As part of the Space Exploration Technology Directorate, a parafoil is proposed for landing on Titan due to its cost effectiveness, ease of deployment, low mass compared to the prospective payload and capabilities of precise autonomous delivery. This paper focuses on path optimization and guidance law development for high-fidelity dynamics parafoil tuning in the dense and adverse wind atmosphere of Titan, defined as a nonlinear and nonconvex optimal control problem. The powerful successive convexification method is used to solve the problem accordingly. The algorithm is designed such that the converged solution adheres to the nonlinear dynamics and kinematics in accordance with the original formulation, while respecting the state and control constraints. The six-degree-of-freedom (6DoF) simulations results show that this robust method is suitable for autonomous interplanetary applications.

\section{Nomenclature}

$\begin{array}{llllll}\alpha & \text { angle-of-attack, rad } & \mathbf{A} & \text { state coefficient matrix } & m & \text { spacecraft mass, } \mathrm{kg} \\ \beta & \text { angle of sideslip, rad } & \mathcal{A} & \text { aerodynamic frame } & \mathbf{M} & \text { moment vector, } \mathrm{N} \cdot \mathrm{m} \\ \gamma_{g s} & \text { glide-slope constraint, } \mathrm{rad} & a & \text { parafoil height, } \mathrm{m} & \mathbf{n}_{i} & i^{\text {th }} \text {-axis unit vector } \\ \delta_{a} & \text { asymmetric deflection, } \mathrm{rad} & \mathbf{B} & \text { control coefficient matrix } & \mathbf{q} & \text { quaternion vector } \\ \delta_{s} & \text { symmetric deflection, } \mathrm{rad} & \mathcal{B} & \text { body frame } & \mathbf{r} & \text { position vector, } \mathrm{m} \\ \epsilon & \text { anhedral angle, rad } & b & \text { parafoil wingspan, } \mathrm{m} & \mathbf{v} & \text { velocity vector, } \mathrm{m} / \mathrm{s} \\ \theta & \text { pointing angle, } \mathrm{rad} & C_{\zeta} & \text { aerodynamic coefficient } \zeta & R & \text { parafoil line length, } \mathrm{m} \\ \mu & \text { rigging angle, rad } & \mathbf{C}_{\mathcal{B} \mid \mathcal{I}} & \text { direction cosine matrix } & S_{\text {ref }} & \text { reference area, } \mathrm{m}^{2} \\ \boldsymbol{v} & \text { virtual control vector } & c & \text { parafoil chord, } \mathrm{m} & t & \text { time, } \mathrm{s} \\ \rho & \text { density, } \mathrm{kg} / \mathrm{m}^{3} & \mathbf{F} & \text { force vector, } \mathrm{N} & t & \text { parafoil thickness, } \mathrm{m} \\ \chi & \text { heading angle, } \mathrm{rad} & f & \text { apparent } & \mathbf{u} & \text { control vector } \\ \tau & \text { normalized trajectory time } & \mathbf{g} & \text { gravity acceleration, } \mathrm{m} / \mathrm{s}^{2} & \mathbf{x} & \text { state vector } \\ \mathbf{\Phi} & \text { state transition matrix } & \mathbf{I} & \text { inertia tensor, } \mathrm{kg} \cdot \mathrm{m}^{2} & {[\cdot \times]} & \text { skew-symmetric } \\ \boldsymbol{\omega} & \text { angular velocity vector, } \mathrm{rad} / \mathrm{s} & \mathcal{I} & \text { inertial frame } & \|\cdot\| & \text { vector norm }\end{array}$

(C) 2020 California Institute of Technology. Government sponsorship acknowledged. This research was carried out at the Jet Propulsion Laboratory, California Institute of Technology, under a contract with the National Aeronautics and Space Administration. Published by the American Institute of Aeronautics and Astronautics, Inc., with permission.

*Fulbright Scholar rayan.mazouz@jpl.nasa.gov

${ }^{\dagger}$ Principal, Group Supervisor, Robotics Modeling \& Simulation Group, marco.b.quadrelli@jpl.nasa.gov Associate Fellow AIAA

$\ddagger$ Associate Professor, Chair Astrodynamics \& Space Missions, e.mooij@tudelft.nl Associate Fellow AIAA. 


\section{Introduction}

$\mathrm{F}$ ROM the perspective of studying prebiotic chemistry, Saturn's largest moon Titan is presumably one of the richest environments in the Solar System. Quadrelli et al. [1] state that studying the science of matter within Titan's atmosphere and beneath its surface is one of the most important planetary science objective. Its geophysical features are such that it is the only moon that has a substantial atmosphere, with a complex chemistry that surpasses any other planetary body in the Solar System [2]. Within the scope op testing hypotheses regarding the development and omnipresence of life in the Universe, Titan may be the ideal planetary body in the Solar System [3]. The wealth of information about Titan and its atmosphere has mainly been obtained from the notable Cassini-Huygens mission mapping the Saturnian system. Huygens was developed to land on Titan, nonetheless it did not employ optimal guidance and control during EDL.

Examining the organic compounds on Titan requires spacecraft to land near regions of fluids and sediments, to be encountered near seas and lakes. Within current technological capabilities landing dispersions extend hundreds of kilometers wide. This precludes landing on large liquid areas, with the exception of the lakes at the northern high latitudes. Due to the seasons of Titan, space landing missions to these northern lakes are prevented prior to the late 2030s. This entails that access to dynamic environments conducive to chemical evolution relies heavily on drift due wind. For these epitomized reasons, precision landing capabilities is an anticipated technology for exploring the habitat of Titan.

The greatest source of landing error for a Titan mission is attributed to high altitude winds, and their respective uncertainties due to lack of exploration. For missions requiring low landing accuracy, a high altitude (about $150 \mathrm{~km}$ ) deployed parachute would suffice. For precise low delivery error however, an unguided drogue parachute is proposed for the initial phase, followed by a deployed guided parafoil at an altitude of 40 $\mathrm{km}$. This altitude has been chosen because descent cameras can view the surface for state estimation purposes, reducing the landing error norm by over $100 \mathrm{~km}$. This implies that for adequate state knowledge, Terrain Relative Navigation (TRN) is of particular need for a Titan mission.

As part of the NASA Space Exploration Technology Directorate, an autonomous Precision Aerial Delivery Systems (PADS) has been chosen to be the payload delivery system. Due to the dense nature of Titan's atmosphere, the nominal landing sequence takes hours. The benefit of this extended EDL sequence is the fact that less strain is put on the GNC systems in terms of computational speed, as time is less of a driving factor within the autonomy framework. A controlled parafoil has several advantages over the traditional parachutes. First and foremost, classical canopies have (almost) no control authority. Systems like PADS however have actuators that allow for remote in-flight control capabilities. This paper is a continuation of earlier work by Quadrelli et al. [1] on Titan aeromaneuvering, Mooij [4] on flight mechanics, and Mazouz [5] on optimal landing, focusing on the terminal descent path optimization. We adapt the parafoil flights mechanics model by Yakimenko [6], and the robust successive convexification method by Szmuk and Acikmese [7] and Szmuk et al. [8] for Mars powered descent guidance. Control authority is obtained by parafoil deflections which alter the aerodynamic forces and moments. The solution process of the nonconvex six-degree-of-freedom (6DoF) optimal control problem is initialized by utilizing a dynamically inconsistent trajectory, made feasible through a virtual control vector. The algorithm is solved in a successive iterative manner and designed such that the converged solution adheres to the nonlinear dynamics and kinematics in accordance with the original formulation, while respecting the state and control constraints. To cope with the requirements of TRN, a pointing constraint is incorporated within the guidance framework. We believe that this robust method is suitable for autonomous interplanetary applications.

The final segment of this introduction outlines the structure of this paper. The formulation of the governing dynamics is outlined in Sec. III Subsequently, in Sec. III. the non-convex formulation is characterized, followed by the method description for transferring the problem to a linear and discrete formulation in Sec. IV] The successive convexification framework is established in Sec. V The nominal and sensitivity analysis results are portrayed and interpreted in Sec. VI] while Sec. VII confers conclusive remarks. 


\section{Dynamics Formulation}

This section outlines the dynamics and associated aerodynamics of the minimum control effort parafoil landing problem through a $6 \mathrm{DoF}$ nonconvex formulation. The structure of this section is as follows. In Sec. II.A the governing spacecraft Equations of Motion (EoM) are conferred. Subsequently, the aerodynamic properties of the parafoil and the computation method of the aerodynamic forces and moments are formulated in Sec. II.B For the formulation of the problem, a number of reference frames are defined:

$\mathcal{A}$ The aerodynamic frame in which the aerodynamic angles are defined: angle-of-attack $\alpha$ and side-slip angle $\beta$. The relative orientation of these angles is depicted in Fig. 1

$\mathcal{B}$ The body frame is fixed to the vehicle. The angular rate of the vehicle, expressed as the vector $\omega$, defines the rotation of the body frame with respect to the inertial frame.

I The inertial frame has its origin coinciding with the landing target. By assuming Titan to be non-rotational and the frame having an Up-East-North orientation, it becomes inertial in nature.

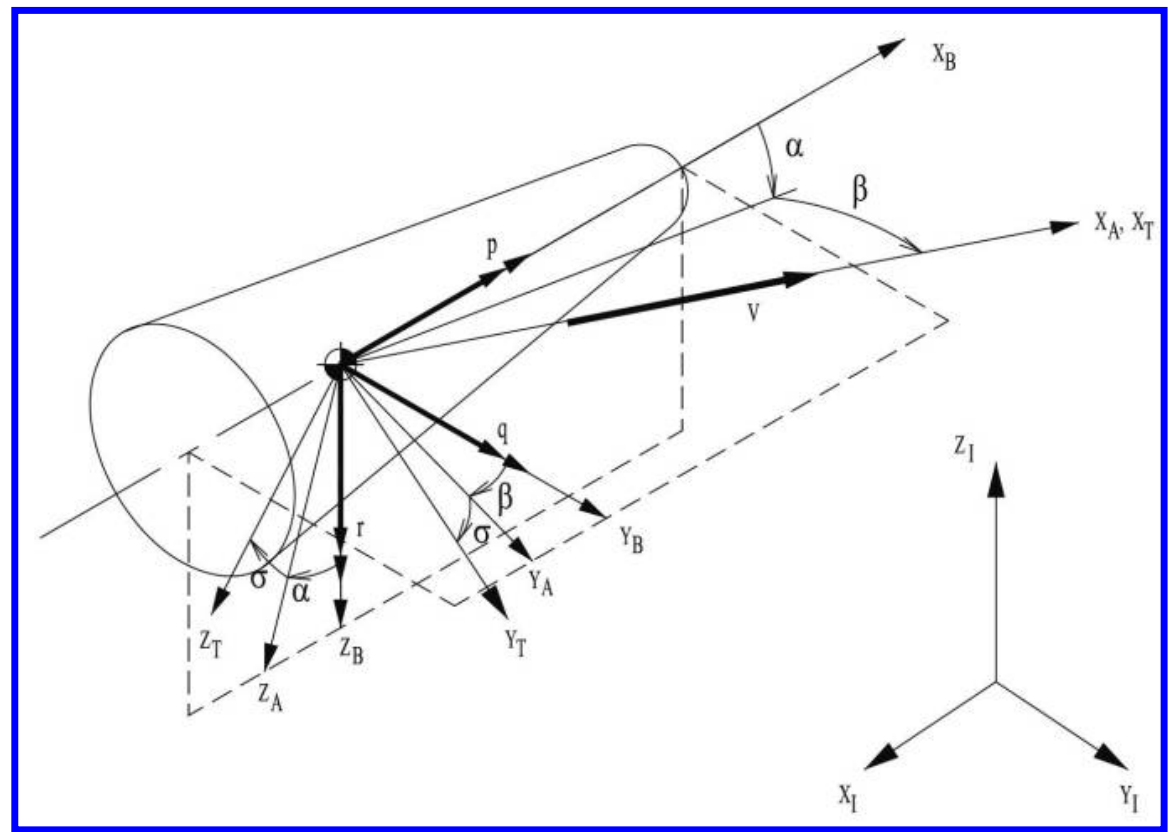

Fig. 1 The (positive) aerodynamic angles $\alpha$ and $\beta$ defining the relation between the body frame $\mathcal{B}$ and the aerodynamic frame $\mathcal{A}[4]$.

Any body moving within a fluid causes that particular fluid to be set into motion which generates a field of pressure about the body. This field is what is known as apparent mass pressure, a ratio between the mass of the vehicle and the shifted mass of air. The dynamic model for this phenomenon is described in Sec. II.C.

\section{A. Spacecraft Dynamics and Kinematics}

The spacecraft is modelled as a rigid body parafoil-payload system, and due to low altitudes subject to a constant gravitational acceleration $\mathbf{g}_{I}$. The system mass $m$ is constant over time, and the total force on the vehicle expressed in $\mathcal{I}$ is defined as $\mathbf{F}_{\mathcal{I}} \in \mathbb{R}^{3}$. Position and velocity are defined in the same coordinate system, denoted by $\mathbf{r}_{I} \in \mathbb{R}^{3}$ and $\mathbf{v}_{\mathcal{I}} \in \mathbb{R}^{3}$, respectively. The equations describing the translational dynamics are:

$$
\begin{aligned}
\dot{\mathbf{r}}_{I}(t) & =\mathbf{v}_{I}(t) \\
\dot{\mathbf{v}}_{\mathcal{I}}(t) & =\frac{\mathbf{F}_{I}(t)}{m}+\mathbf{g}_{I}
\end{aligned}
$$


A unit quaternion attitude representation is adapted due its beneficial singularity-free nature. The spacecraft attitude in the $\mathcal{B}$-frame relative to the $\mathcal{I}$-frame is denoted as $\mathbf{q}_{\mathcal{B} \mid \mathcal{I}} \in \mathbb{S}^{3}$ :

$$
\mathbf{q}_{\mathcal{B} \mid \mathcal{I}}(t)=\left(\begin{array}{c}
\cos \frac{\zeta}{2} \\
\sin \left(\frac{\zeta}{2}\right) \hat{\mathbf{n}}
\end{array}\right)=\left(\begin{array}{llll}
q_{1} & q_{1} & q_{2} & q_{4}
\end{array}\right)^{T}
$$

Notice that this parameterization is established such that the attitude has three vector components that reside on a three-dimensional sphere $\mathbb{S}^{3}$, which through a scalar part is embedded in four-dimensional Euclidean space [9]. The Direction Cosine Matrix (DCM) $\mathbf{C}_{I, \mathcal{B}}$ encompasses the transformation of the quaternion attitude from the $\mathcal{B}$-frame to the $\mathcal{I}$-frame, for which the relation is provided by:

$$
\mathbf{C}_{\mathcal{I}, \mathcal{B}}=\left[\begin{array}{ccc}
q_{1}^{2}+q_{1}^{2}-q_{2}^{2}-q_{4}^{2} & 2\left(q_{1} q_{2}-q_{1} q_{4}\right) & 2\left(q_{1} q_{4}+q_{1} q_{2}\right) \\
2\left(q_{1} q_{2}+q_{1} q_{4}\right) & q_{1}^{2}-q_{1}^{2}+q_{2}^{2}-q_{4}^{2} & 2\left(q_{2} q_{4}-q_{1} q_{1}\right) \\
2\left(q_{1} q_{4}-q_{1} q_{2}\right) & 2\left(q_{2} q_{4}+q_{1} q_{1}\right) & q_{1}^{2}-q_{1}^{2}-q_{2}^{2}+q_{4}^{2}
\end{array}\right]
$$

Utilizing the DCM, the force $\mathbf{F}_{\mathcal{I}}$ is computed based on the total force in the $\mathcal{B}$-frame. The force $\mathbf{F}_{\mathcal{B}}$ has multiple contributing sources, all of which are of an aerodynamic nature.

$$
\mathbf{F}_{\mathcal{I}}=\mathbf{C}_{I, \mathcal{B}} \mathbf{F}_{\mathcal{B}}
$$

In Section II.B each individual $\mathbf{F}_{i}$ contributor is outlined, including the methodology for transforming these forces to the $\mathcal{B}$-frame. The generalized relation is defined as:

$$
\mathbf{F}_{\mathcal{B}}=\sum_{i=1}^{n} \mathbf{F}_{i}
$$

We define a vector $\boldsymbol{\omega}_{\mathcal{B}} \in \mathbb{R}^{3}$ to quantify the angular velocity of the $\mathcal{B}$-frame around the $\mathcal{I}$-frame. The angular velocity skew-symmetric matrices $\left[\boldsymbol{\omega}_{\mathcal{B}} \times\right]$ and $\Omega\left(\boldsymbol{\omega}_{\mathcal{B}}\right)$ are defined as:

$$
\left[\boldsymbol{\omega}_{\mathcal{B}} \times\right] \triangleq\left[\begin{array}{ccc}
0 & -\omega_{z} & \omega_{y} \\
\omega_{z} & 0 & -\omega_{x} \\
-\omega_{y} & \omega_{x} & 0
\end{array}\right] \quad \Omega\left(\boldsymbol{\omega}_{\mathcal{B}}\right) \triangleq\left[\begin{array}{cccc}
0 & -\omega_{x} & -\omega_{y} & -\omega_{z} \\
\omega_{x} & 0 & \omega_{z} & -\omega_{y} \\
\omega_{y} & -\omega_{z} & 0 & \omega_{x} \\
\omega_{z} & \omega_{y} & -\omega_{x} & 0
\end{array}\right]
$$

The total moment on the spacecraft $\mathbf{M}_{\mathcal{B}} \in \mathbb{R}^{3}$ and the inertia tensor $\mathbf{I}_{\mathcal{B}}$ are established in the $\mathcal{B}$-frame, which are needed to compute the attitude dynamics and quaternion kinematics, respectively:

$$
\begin{aligned}
\mathbf{I}_{\mathcal{B}} \dot{\boldsymbol{\omega}}_{\mathcal{B}}(t) & =\mathbf{M}_{\mathcal{B}}-\left[\boldsymbol{\omega}_{\mathcal{B}} \times\right] \mathbf{I}_{\mathcal{B}} \boldsymbol{\omega}_{\mathcal{B}} \\
\dot{\mathbf{q}}_{\mathcal{B} \mid \mathcal{I}}(t) & =\frac{1}{2} \Omega\left(\boldsymbol{\omega}_{\mathcal{B}}\right) \mathbf{q}_{\mathcal{B} \mid \mathcal{I}}
\end{aligned}
$$

For the landing sequence it is assumed that the Center-of-Mass (CoM) of the parafoil-payload system remains constant, from which follows that the moment arm to the aerodynamic center is time-invariant. This distance $\mathbf{r}_{\mathbf{F}, \mathcal{B}} \in \mathbb{R}^{3}$ is defined in the $\mathcal{B}$-frame and the moment torque $\mathbf{M}_{\mathbf{F}}$ it causes on the system is computed through Eq. 7. Notice that $\left[\mathbf{r}_{\mathbf{F}, \mathcal{B}} \times\right]$ is a skew-symmetric matrix.

$$
\mathbf{M}_{\mathbf{F}, \mathcal{B}}=\left[\mathbf{r}_{\mathbf{F}, \mathcal{B}} \times\right] \mathbf{F}_{\mathcal{B}}
$$

The total moment $\mathbf{M}_{\mathcal{B}}$ is likewise obtained by adding the individual contributors $\mathbf{M}_{i}$, such that:

$$
\mathbf{M}_{\mathcal{B}}=\sum_{i=1}^{n} \mathbf{M}_{i}
$$




\section{B. Aerodynamics}

The nominal aerodynamic forces and moments, i.e., without considering wind and apparent aerodynamic phenomena yet, derived in this section are based on the high-fidelity 6DoF rigid-body model depicted in Fig. 2 The control authority for a parafoil is related to controlling the aerodynamic forces and moments. In this paper, the model is designed such that the symmetric deflection $\delta_{s}$ controls the longitudinal dynamics, while asymmetric deflection $\delta_{a}$ controls the lateral dynamics. These two parameters are a function of the canopy's right and left deflection, $\delta_{r}$ and $\delta_{l}$, respectively:

$$
\begin{aligned}
\delta_{s} & =\frac{1}{2}\left(\delta_{r}+\delta_{l}\right) \\
\delta_{a} & =\delta_{r}-\delta_{l}
\end{aligned}
$$

We start by deriving the aerodynamic forces, drag, side force and lift, denoted as $D, S$ and $L$, respectively. We define the local dynamic pressure $\bar{q}=\frac{1}{2} \rho V_{a}^{2}$, and $S_{\text {ref }}$ as the reference area of the vehicle. The system has two contributors, which are the parafoil and the payload. To ease the discussion, these two contributors are given subscripts 1 and 2, respectively. The general relation for the aerodynamic force is:

$$
\mathbf{F}_{\mathcal{A}}=\left(\begin{array}{c}
-D \\
-S \\
-L
\end{array}\right)=\bar{q} S_{\mathrm{ref}}\left(\begin{array}{c}
-C_{D} \\
-C_{S} \\
-C_{L}
\end{array}\right)
$$

The total drag of the parafoil is increased by $30 \%$ to account for the contribution of the system suspension lines [5]. The coefficients for the parafoil are computed through:

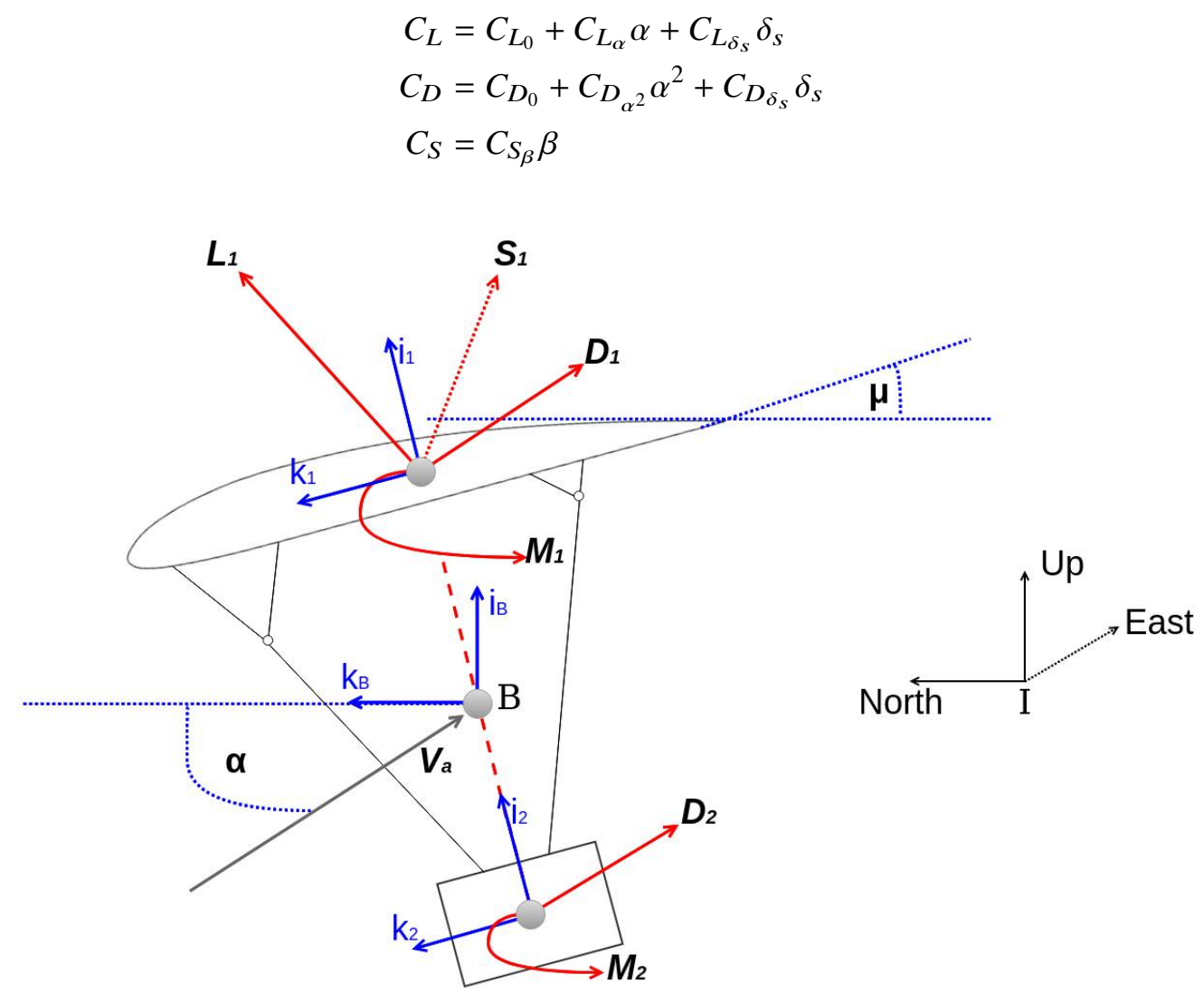

Fig. 2 Side view of the 6DoF parafoil-payload system subject to aerodynamic forces and moments. 
It becomes evident that the coefficients are a function of the before-mentioned symmetric deflection, angle-of-attack and side-slip angle. The latter two parameters are computed through:

$$
\alpha=\tan ^{-1}\left(\frac{w}{u}\right) \quad \beta=\tan ^{-1}\left(\frac{v}{\sqrt{u^{2}+w^{2}}}\right)
$$

The velocity components are based on the local air velocity $V_{a}=(u, v, w)^{T}$. In Table 1 the aerodynamic force coefficients are provided. The payload only contributes to the total drag value, with $C_{D_{2}}=1.0$. As the aerodynamic relations are defined in the $\mathcal{A}$-frame, a transformation to the $\mathcal{B}$-frame is required:

$$
\mathbf{F}_{\mathcal{B}}=\mathbf{C}_{\mathcal{B}, \mathcal{A}} \mathbf{F}_{\mathcal{A}}
$$

in which the transformation matrix $\mathbf{C}_{\mathcal{B}, \mathcal{A}}$ is computed by means of the transpose of the following relation:

$$
\mathbf{C}_{\mathcal{A}, \mathcal{B}}=\mathbf{C}_{\mathcal{B}, \mathcal{A}}^{T}=\left[\begin{array}{ccc}
\cos \alpha \cos \beta & -\sin \beta & \sin \alpha \cos \beta \\
\cos \alpha \sin \beta & \cos \beta & \sin \alpha \sin \beta \\
-\sin \alpha & 0 & \cos \alpha
\end{array}\right]
$$

We remark anew that there are two sources which have a contributing factor to the aerodynamic force in the $\mathcal{B}$-frame: the parafoil aerodynamic force $\mathbf{F}_{1}=\left(L_{1}, S_{1}, D_{1}\right)^{T}$ and the payload aerodynamic force $\mathbf{F}_{2}=\left(0,0, D_{2}\right)^{T}$, as depicted in Fig. 2. The total nominal aerodynamic force hence equals:

$$
\mathbf{F}_{\mathcal{B}}=\mathbf{F}_{1}+\mathbf{F}_{2}
$$

Table 1 Properties for modeling the aerodynamic forces, adjusted accordingly based on [1] and [6]. This table contains the most up-to-date design parameters.

\begin{tabular}{lll}
\hline \hline Parameters & Parafoil & Payload \\
\hline \multirow{3}{*}{ Lift coefficients } & $C_{L, 0}=0.091$ & \\
& $C_{L, \alpha}=0.90 \quad-$ \\
& $C_{L, \delta_{s}}=0.21$ & \\
\hline \multirow{3}{*}{ Drag coefficients } & $C_{D, 0}=0.25$ & \\
& $C_{L, \alpha^{2}}=0.12$ & $C_{D_{2}}=1.0$ \\
& $C_{L, \delta_{s}}=0.30$ & \\
\hline Side force coefficient & $C_{S, \beta}=-0.23$ & - \\
\hline \hline
\end{tabular}

In a similar fashion, the aerodynamic moments are derived in Eq. 18 roll, pitch and yaw, denoted as $\mathcal{L}, \mathcal{M}$ and $\mathcal{N}$, respectively. For this the mater the parafoil wingspan $b$ and chord $c$ are required, such that:

$$
\mathbf{M}_{\mathcal{A}}=\left(\begin{array}{c}
\mathcal{L} \\
\mathcal{M} \\
\mathcal{N}
\end{array}\right)=\bar{q} S_{\text {ref }}\left(\begin{array}{c}
C_{l} b \\
C_{m} c \\
C_{n} b
\end{array}\right)
$$

In accordance with the Up-East-North convention, $(p, q, r)^{T}=\left(\omega_{z}, \omega_{y}, \omega_{x}\right)^{T}$. This relation is needed to compute the moment coefficients, based on the following set of equations:

$$
\begin{aligned}
C_{l} & =C_{l_{\beta}} \beta+C_{l_{\delta_{a}}} \delta_{a}+C_{l_{p}} \frac{b}{2 V} p+C_{l_{r}} \frac{b}{2 V} r \\
C_{m} & =C_{m_{0}}+C_{m_{\alpha}} \alpha+C_{m_{q}} \frac{c}{2 V} q \\
C_{n} & =C_{n_{\beta}} \beta+C_{n_{\delta_{a}}} \delta_{a}+C_{n_{p}} \frac{b}{2 V} p+C_{n_{r}} \frac{b}{2 V} r
\end{aligned}
$$


The two moments $\mathbf{M}_{1}$ and $\mathbf{M}_{2}$ can now also be quantified. We define vectors $\mathbf{r}_{1}$ and $\mathbf{r}_{2}$, which denote the Euclidean space distance from the system CoM to the point of application of the aerodynamic force of the parafoil and payload, respectively. From this we derive that the total parafoil moment contribution equals:

$$
\mathbf{M}_{1}=\left[\mathbf{r}_{1} \times\right] \mathbf{F}_{1}+\mathbf{M}_{\mathcal{A}}
$$

It is assumed that only the parafoil has contributing moment derivatives, and hence the payload only contributes to the moment $\mathbf{M}_{2}$ due to the $\operatorname{drag} D_{2}$, such that:

$$
\mathbf{M}_{2}=\left[\mathbf{r}_{2} \times\right] \mathbf{F}_{2}
$$

Through Eq. 8, the total nominal moment is computed by means of a summation. In Table 2 the magnitudes of the aerodynamic parafoil moment coefficients are provided.

Table 2 Properties for modeling the aerodynamic moments, adjusted accordingly based on [1].

\begin{tabular}{llll}
\hline \hline Parameters & Roll Coefficients & Pitch Coefficients & Yaw Coefficients \\
\hline \multirow{4}{*}{ Parafoil } & $C_{l, \beta}=-0.036$ & $C_{m, 0}=0.25$ & $C_{n, \beta}=-0.0015$ \\
& $C_{l, \delta_{a}}=-0.0035$ & $C_{m, \alpha}=-0.72$ & $C_{n, \delta_{a}}=0.0155$ \\
& $C_{l, p}=-0.84$ & $C_{m, q}=-1.49$ & $C_{n, p}=-0.082$ \\
& $C_{l, r}=-0.082$ & & $C_{n, r}=-0.27$ \\
\hline
\end{tabular}

As part of the $6 \mathrm{DoF}$ model, the moments of inertia need to be computed. Starting with the parafoil case, in which the mass denotes the parafoil mass. The canopy volume is approximated based on Yakimenko [6] to equal $v_{\mathrm{vol}}=0.09 c^{2}$. The inertia tensor for the parafoil is computed by:

$$
\mathbf{I}_{1}=\left[\begin{array}{ccc}
\frac{v_{\mathrm{vol}} \rho+m_{1}}{12}\left(b_{\mathrm{inf}}^{2}+{\frac{v_{\mathrm{vol}}}{c b}}^{2}\right) & 0 & 0 \\
0 & \frac{v_{\mathrm{vol}} \rho+m_{1}}{12}\left(c^{2}+{\frac{v_{\mathrm{vol}}}{c b}}^{2}\right) & 0 \\
0 & 0 & \frac{v_{\mathrm{vol}} \rho+m_{1}}{12}\left(b_{\mathrm{inf}}^{2}+c^{2}\right)
\end{array}\right]
$$

In the above equation $\rho$ defines the planetary body atmospheric density. Furthermore, we define the anhedral angle $\epsilon=\frac{b}{2 R}$, with $R$ being the length of the suspension lines. From these two parameters follows the inflated wingspan $b_{\text {inf }}=2 R \cdot \sin \epsilon$. As the parafoil is inclined by the rigging angle, as depicted in Fig. 2, the inertia tensor must be transformed to the $\mathcal{B}$-frame, which is established by:

$$
\mathbf{I}_{1, \mathcal{B}}=\mathbf{C}_{1, \mathcal{B}} \mathbf{I}_{1} \mathbf{C}_{1, \mathcal{B}}^{T} \quad \text { with } \quad \mathbf{C}_{1, \mathcal{B}}=\left[\begin{array}{ccc}
\cos \mu & 0 & \sin \mu \\
0 & 1 & 0 \\
-\sin \mu & 0 & \cos \mu
\end{array}\right]
$$

For the payload a box is assumed with equal dimensions in all directions, such that the inertia tensor $\mathbf{I}_{2}$ is obtained through:

$$
\mathbf{I}_{2}=\left[\begin{array}{ccc}
\frac{m_{2}}{12}\left(b_{2}^{2}+c_{2}^{2}\right) & 0 & 0 \\
0 & \frac{m_{2}}{12}\left(a_{2}^{2}+c_{2}^{2}\right) & 0 \\
0 & 0 & \frac{m_{2}}{12}\left(a_{2}^{2}+b_{2}^{2}\right)
\end{array}\right]
$$

The magnitudes of the parameters utilized to calculate the magnitudes of the aerodynamic properties are in accordance with Tables 1 and 4 of Quadrelli et al. [1], with an exception for the parafoil surface area (the updated value has been included in Table 3). This concludes the section on aerodynamics. The next segment outlines the apparent inertia and moment generated due pressure caused by a body set in motion in a fluid. 


\section{Apparent Aerodynamics}

Even tough the nominal mass of a parafoil is assumed to be constant over time, apparent phenomena cause changes in motion behavior, adding to the total moment and inertia. Before continuing the discussion on the convex guidance problem, the definition and mathematical principles of apparent masses and inertias are outlined, primarily based on Lissaman and Brown [10]. Any body moving within a fluid causes that particular fluid to be set into motion which generates a field of pressure about the body. For an aircraft this phenomena is often neglected, however, for a parafoil it heavily influences the ram-air dynamics characteristics. This ratio $M_{r}$ is approximated through [10]:

$$
M_{r}=\frac{m}{\rho S_{\text {ref }}^{2 / 3}}
$$

The density of Titan's atmosphere is approximated by the Yelle model [11], defined as:

$$
\rho=5.43 e^{-0.512 \cdot 10^{-3} \cdot h}
$$

Notice that the density is a function of altitude, which is a function of time. A ratio $M_{r}$ in the order of 0.8 is typical for Earth-like environments, while a parafoil within the Titan environment typically has values of 7. In Figs. 3 and 4 a geometric visualization is provided of an inflated canopy with apparent masses and inertias. The computed mass and inertia are not the real dynamic fluid properties but a representation of the transported energy due acceleration, causing additional work which increases the velocity [12]. The tensors of the apparent mass and inertia are defined as:

$$
\mathbf{M}_{f}=\left[\begin{array}{lll}
A & 0 & 0 \\
0 & B & 0 \\
0 & 0 & C
\end{array}\right] \text { and } \quad \mathbf{I}_{f}=\left[\begin{array}{ccc}
I_{A} & 0 & 0 \\
0 & I_{B} & 0 \\
0 & 0 & I_{C}
\end{array}\right]
$$

The individual components of these governing equations are computed by means of the two set of equations given in Eqs. (29) and (30). In these equations $A R$ is the aspect ratio, $b$ the wingspan of the parafoil, $c$ the parafoil chord, $h$ the parafoil height and $t$ its thickness. Likewise, these parameters have magnitudes in accordance with Tabs. 1 and 4 of Quadrelli et al. [1]. For the purpose of convenience, the equations are
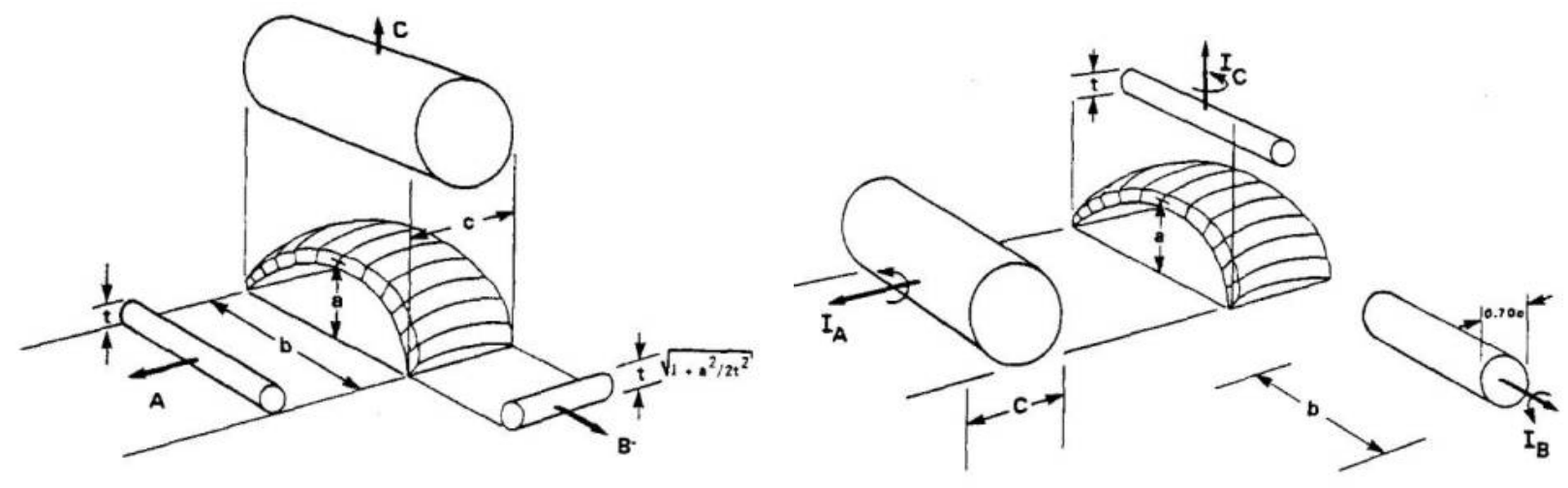

Fig. 3 Depiction of the apparent mass due to a parafoil in an aerodynamic environment [10].
Fig. 4 Depiction of the apparent inertia due to a parafoil in an aerodynamic environment [10]. 
related through $A R=b / c, t_{\star}=t / c$ and $a_{\star}=a / b$, such that the following relations are established:

$$
\begin{aligned}
A & =0.667 \rho\left(1+\frac{8}{3} a_{\star}^{2}\right) t^{2} b \\
B & =0.267 \rho\left(1+2 \frac{a_{\star}^{2}}{t_{\star}^{2}} A R\left(1-t_{\star}^{2}\right) t^{2} c\right. \\
C & =0.785 \rho \sqrt{1+2+a_{\star}\left(1-t_{\star}\right)} \frac{A R}{1+A R} c^{2} b \\
I_{A} & =0.055 \rho \frac{A R}{1+A R} c^{2} b^{3} \\
I_{B} & =0.0308 \rho \frac{A R}{1+A R}\left[1+\frac{\pi}{6}(1+A R) A R a_{\star}^{2} t_{\star}^{2}\right] c^{4} b \\
I_{C} & =0.0555 \rho\left(1+8 a_{\star}^{2}\right) t^{2} b^{3}
\end{aligned}
$$

The apparent mass in $z$-direction is the greatest contributor, specifically, the same order of magnitude as the entire canopy. This means the contribution is still significantly lower in order of magnitude compared to the payload, nonetheless, significant enough to be considered. Furthermore, the apparent inertia is largest in $y$-direction for short suspension lines. The contribution of the apparent inertia is likewise in the same order of magnitude as the canopy. Now that the matter on varying mass has been outlined, the discussion on the dynamics formulation is concluded. The next section conveys the nonconvex problem.

\section{Non-Convex Formulation}

In this section, we define the parafoil landing problem as a non-convex optimization problem in continuoustime space. To allow the optimal control problem to be solved, we apply the successive convexification method, followed by a linearization and discretization process in the subsequent section. Distinct from powered descent guidance, the control authority is provided through the electric powered pull of the landing canopy. The algorithm is formulated as a minimum control effort problem:

$$
\begin{aligned}
& 0<F_{\mathcal{A}, \text { min }} \leq\left\|\mathbf{F}_{\mathcal{A}}\right\|_{2} \leq F_{\mathcal{A}, \text { max }} \quad \rightarrow \quad \text { subject to: } 0 \leq \delta_{s} \leq 1 \\
& 0<M_{\mathcal{A}, \text { min }} \leq\left\|\mathbf{M}_{\mathcal{A}}\right\|_{2} \leq M_{\mathcal{A}, \text { max }} \quad \rightarrow \quad \text { subject to: } 0 \leq \delta_{a} \leq 1
\end{aligned}
$$

These aerodynamic parameters drive the motion of the parafoil. As an absolute zero magnitude of neither the aerodynamic force nor the moment can be guaranteed, the problem becomes non-convex in nature. The cost function is defined as the minimization of the control effort during time-of-flight $t_{f}$ :

$$
\min _{t_{f}}\|\mathbf{u}(t)\|
$$

The glide-slope constraint enforces that a specific optimal trajectory to the target is not too shallow nor goes below the surface. It requires the spacecraft to remain within a cone as defined by the minimum slope angle. To restrict the vehicle motion accordingly, the glide-slope angle $\gamma_{g s}$ is imposed with its center at the origin of the $\mathcal{I}$-frame. The convex cone constraint is imposed as follows:

$$
\mathbf{n}_{1} \cdot \mathbf{r}_{\mathcal{I}} \geq \tan \gamma_{g s}\left\|H_{23}^{T} \mathbf{r}_{\mathcal{I}}\right\|_{2} \quad \text { with } \quad H_{23} \triangleq\left[\begin{array}{ll}
\mathbf{n}_{2} & \mathbf{n}_{3}
\end{array}\right]
$$

To cope with the requirement of TRN, the spacecraft orientation in terms of attitude is restrained. The tilt angle $\theta$ is defined as the angle between the $\mathrm{X}$-axes of the $\mathcal{B}$ and $\mathcal{I}$, such that:

$$
\cos \theta=\mathbf{n}_{1} \cdot C_{\mathcal{I}, \mathcal{B}} \mathbf{n}_{1}=1-2\left(q_{2}^{2}+q_{3}^{2}\right)
$$


A maximum $\theta_{\max }$ is imposed to avoid excessive tilt, with the convex constraint formulated as:

$$
\cos \theta_{\max } \leq 1-2\left(q_{2}^{2}+q_{3}^{2}\right)
$$

The angle-of-attack $\alpha$ is constrained to a threshold maximum value to avoid stall through:

$$
\mathbf{v} \cdot \mathbf{n}_{2} \leq \tan \left(\alpha_{\max }\right) \mathbf{v} \cdot \mathbf{n}_{1}
$$

Defining the aerodynamic angles as states rather than control variables implies that they are assumed to be a consequence of motion. Furthermore, a maximum angular rate $\omega_{\max }$ convex constraint is imposed as:

$$
\left\|\omega_{B}\right\|_{2} \leq \omega_{\max }
$$

The boundary conditions of the sequence are constrained such that the soft-landing is enforced through zero position deviation (with respect to the target in the guidance frame) and zero touchdown velocity at termination in three-dimensional Euclidean space. The initial attitude is considered to be nominal, and constrained to be zero at termination. The complete non-convex continuous-time problem description for minimum control effort $6 \mathrm{DoF}$ landing guidance is provided in Problem 1 .

\section{Problem 1: Non-convex continuous time minimum control effort guidance problem}

Cost Function:

$$
\min _{t_{f}}\|\mathbf{u}(t)\| \quad \text { subject to: }
$$

Dynamics:

$$
\begin{aligned}
\dot{\mathbf{r}}_{\mathcal{I}}(t) & =\mathbf{v}_{\mathcal{I}}(t) \\
\dot{\mathbf{v}}_{\mathcal{I}}(t) & =\frac{\mathbf{F}_{\mathcal{I}}(t)}{m}+\mathbf{g}_{\mathcal{I}} \\
\mathbf{I}_{\mathcal{B}} \dot{\boldsymbol{\omega}}_{\mathcal{B}}(t) & =\mathbf{M}_{\mathcal{B}}-\left[\boldsymbol{\omega}_{\mathcal{B}} \times\right] \mathbf{I}_{\mathcal{B}} \boldsymbol{\omega}_{\mathcal{B}} \\
\dot{\mathbf{q}}_{\mathcal{B} \mid \mathcal{I}}(t) & =\frac{1}{2} \Omega\left(\boldsymbol{\omega}_{\mathcal{B}}\right) \mathbf{q}_{\mathcal{B} \mid \mathcal{I}}
\end{aligned}
$$

State Constraints:

$$
\begin{aligned}
\tan \gamma_{g s}\left\|H_{23}^{T} \mathbf{r}_{I}\right\|_{2} & \leq \mathbf{n}_{1} \cdot \mathbf{r}_{\mathcal{I}} \\
\cos \theta_{\max } & \leq 1-2\left(q_{2}^{2}+q_{3}^{2}\right) \\
\left\|\boldsymbol{\omega}_{B}\right\|_{2} & \leq \omega_{\max } \\
\mathbf{v} \cdot \mathbf{n}_{2} & \leq \tan \left(\alpha_{\max }\right) \mathbf{v} \cdot \mathbf{n}_{1}
\end{aligned}
$$

Control Constraints:

$$
\begin{array}{rlll}
0<F_{\mathcal{A}, \text { min }} \leq\left\|\mathbf{F}_{\mathcal{A}}\right\|_{2} \leq F_{\mathcal{A}, \text { max }} & \rightarrow & \text { subject to: } & 0 \leq \delta_{s} \leq 1 \\
0<M_{\mathcal{A}, \text { min }} \leq\left\|\mathbf{M}_{\mathcal{H}}\right\|_{2} \leq M_{\mathcal{A}, \text { max }} & \rightarrow & \text { subject to: } & 0 \leq \delta_{a} \leq 1
\end{array}
$$

Boundary Conditions:

$$
\begin{aligned}
\mathbf{r}_{\mathcal{I}}(0) & =\mathbf{r}_{\mathcal{I}, \mathbf{0}}, \quad \mathbf{v}_{\mathcal{I}}(0)=\mathbf{v}_{\mathcal{I}, \mathbf{0}}, \quad \boldsymbol{\omega}_{\mathcal{B}}(0)=\boldsymbol{\omega}_{\mathcal{B}, 0}, \quad \mathbf{q}_{\mathcal{B} / \mathcal{I}}(0)=\left[\begin{array}{llll}
1 & 0 & 0 & 0
\end{array}\right]^{T} \\
\mathbf{r}_{\mathcal{I}}\left(t_{f}\right) & =\mathbf{0}, \quad \mathbf{v}_{\mathcal{I}}\left(t_{f}\right)=\mathbf{0}, \quad \boldsymbol{\omega}_{\mathcal{B}}\left(t_{f}\right)=\mathbf{0}, \quad \mathbf{q}_{\mathcal{B} / \mathcal{I}}\left(t_{f}\right)=\left[\begin{array}{llll}
1 & 0 & 0 & 0
\end{array}\right]^{T}
\end{aligned}
$$




\section{Linearization and Discretization}

This section initiates with outlining linearization theory applied to the parafoil descent guidance problem. Such linearizations are often conducted using Taylor series approximations. Through the linearization it becomes possible to define the program as a convex Second-Order Cone Programming (SOCP) sub-problem. The second part of this section focuses on the discretization of the problem to transfer the problem from continuous-time to a finite-dimensional parameter optimization problem. The linearized and discretized sub-problems are solved in an iterative manner.

\section{A. Linearization}

The nonlinear system is approximated using a first order Taylor series expansion, resulting in a set of coupled differential equations in state space form:

$$
\Delta \mathbf{x}=\mathbf{A} \Delta \mathbf{x}+\mathbf{B} \Delta \mathbf{u}
$$

The matrices $\mathbf{A}$ and $\mathbf{B}$ define the system and input, respectively. These can be derived straight from the nonlinear EoM:

$$
\mathbf{A}=\left[\begin{array}{ccc}
\frac{\partial f_{1}}{\partial x_{1}} & \cdots & \frac{\partial f_{1}}{\partial x_{n}} \\
\vdots & \vdots & \vdots \\
\frac{\partial f_{n}}{\partial x_{1}} & \cdots & \frac{\partial f_{n}}{\partial x_{n}}
\end{array}\right] \quad \text { and } \quad \mathbf{B}=\left[\begin{array}{ccc}
\frac{\partial f_{1}}{\partial u_{1}} & \cdots & \frac{\partial f_{1}}{\partial u_{n}} \\
\vdots & \vdots & \vdots \\
\frac{\partial f_{n}}{\partial u_{1}} & \cdots & \frac{\partial f_{n}}{\partial u_{n}}
\end{array}\right]
$$

Notice that the functions $f_{n}$ represent the various governing EoM that are applied by the guidance system to assure planetary precision landing through the ruling dynamics. This linearization theory is applied to the non-convex problem that has been defined in Sec. III The discussion is initiated with defining the state vector $\mathbf{x}(t) \in \mathbb{R}^{13}$ and control vector $\mathbf{u}(t) \in \mathbb{R}^{6}$ :

$$
\begin{aligned}
& \mathbf{x}(t)=\left[\begin{array}{llll}
\mathbf{r}_{\mathcal{I}}^{T} & \mathbf{V}_{\mathcal{I}}^{T} & \mathbf{q}_{\mathcal{B} / \mathcal{I}}^{T} & \boldsymbol{\omega}_{\mathcal{I}}^{T}
\end{array}\right]^{T} \\
& \mathbf{u}(t)=\left[\begin{array}{ll}
\mathbf{F}_{\mathcal{A}} & \mathbf{M}_{\mathcal{A}}
\end{array}\right]^{T}
\end{aligned}
$$

Recalling the dynamics and kinematics now expressed as a nonlinear vector function $f: \mathbb{R}^{13} \times \mathbb{R}^{6} \rightarrow \mathbb{R}^{13}$ based on the state and control vectors:

$$
\mathbf{x}(t)=f(\mathbf{x}, \mathbf{u})=\left[\begin{array}{llll}
\mathbf{r}_{\mathcal{I}}^{T} & \mathbf{V}_{\mathcal{I}}^{T} & \mathbf{q}_{\mathcal{B} / \mathcal{I}}^{T} & \dot{\boldsymbol{\omega}}_{B}^{T}
\end{array}\right]^{T}
$$

It is the right-hand side of this equation that is approximated using Taylor series of first order with respect to normalized time $\tau \in[0,1]$. For a certain reference trajectory, the state and control are defined as $\hat{\mathbf{x}}(\tau)$ and $\hat{\mathbf{u}}(\tau)$ respectively. At a later stage of the discussion these quantities are construed. The complete linearized formulation normalized with respect to time reads:

$$
\begin{aligned}
\mathbf{x}^{\prime}(\tau) & =\mathbf{A}(\tau) \mathbf{x}(\tau)+\mathbf{B}(\tau) \mathbf{u}(\tau)+\mathbf{z}(\tau) \\
\mathbf{A}(\tau) & =\left.\frac{\partial}{\partial \mathbf{x}} f(\mathbf{x}, \mathbf{u})\right|_{\hat{\mathbf{x}}(\tau), \hat{\mathbf{u}}(\tau)} \\
\mathbf{B}(\tau) & =\left.\frac{\partial}{\partial \mathbf{u}} f(\mathbf{x}, \mathbf{u})\right|_{\hat{\mathbf{x}}(\tau), \hat{\mathbf{u}}(\tau)} \\
\mathbf{z}(\tau) & =-\mathbf{A}(\tau) \hat{\mathbf{x}}(\tau)-\mathbf{B}(\tau) \hat{\mathbf{u}}(\tau)
\end{aligned}
$$




\section{B. Discretization}

The rest of this section discusses the discretization of the optimization problem that is needed to parameterize the continuous time elements into finite dimensions. The following two sets are defined:

$$
\begin{aligned}
& \mathcal{K}=\{0,1, \ldots, K-2, K-1\} \\
& \overline{\mathcal{K}}=\{0,1, \ldots, K-3, K-2\}
\end{aligned}
$$

For the time an index $k$ is introduced such that for all $k \in K$ the normalized time follows from:

$$
\tau_{k}=\left(\frac{k}{K-1}\right)
$$

A hold of first-order is assumed over all time $\tau \in\left[\tau_{k}, \tau_{k+1}\right]$ elements for the preservation of feasibility, such that for all $k \in \bar{K}$ the following control law holds:

$$
\mathbf{u}(\tau)=\left(\frac{\tau_{k+1}-\tau}{\tau_{k+1}-\tau_{k}}\right) \mathbf{u}_{k}+\left(\frac{\tau-\tau_{k}}{\tau_{k+1}-\tau_{k}}\right) \mathbf{u}_{k+1}
$$

For the propagation of the state $\mathbf{x}_{k}=\mathbf{x}\left(\tau_{k}\right)$ to $\mathbf{x}_{k+1}=\mathbf{x}\left(\tau_{k+1}\right)$ a state transition matrix is introduced that is governed through the following differential equation for all $k \in \bar{K}$ :

$$
\frac{d}{d \tau} \boldsymbol{\Phi}_{A}\left(\tau, \tau_{k}\right)=\mathbf{A}(\tau) \boldsymbol{\Phi}_{A}\left(\tau, \tau_{k}\right) \quad \text { with } \quad \boldsymbol{\Phi}_{A}\left(\tau_{k}, \tau_{k}\right)=I
$$

The complete set of governing discrete-time dynamic equations relating $\mathbf{x}_{k}$ to $\mathbf{x}_{k+1}$ for all $k \in \bar{K}$ is:

$$
\begin{aligned}
\mathbf{x}_{k+1} & =\overline{\mathbf{A}}_{k} \mathbf{x}_{k}+\overline{\mathbf{B}}_{k} \mathbf{u}_{k}+\overline{\mathbf{C}}_{k} \mathbf{u}_{k+1}+\overline{\mathbf{z}}_{k} \\
\overline{\mathbf{A}}_{k} & =\boldsymbol{\Phi}_{A}\left(\tau_{k+1}, \tau_{k}\right) \\
\overline{\mathbf{B}}_{k} & =\int_{\tau_{k}}^{\tau_{k+1}} \boldsymbol{\Phi}_{A}\left(\tau_{k+1}, \xi\right) \mathbf{B}(\xi) \alpha_{k}(\xi) d \xi \\
\overline{\mathbf{C}}_{k} & =\int_{\tau_{k}}^{\tau_{k+1}} \boldsymbol{\Phi}_{A}\left(\tau_{k+1}, \xi\right) \mathbf{B}(\xi) \beta_{k}(\xi) d \xi \\
\bar{z}_{k} & =\int_{\tau_{k}}^{\tau_{k+1}} \boldsymbol{\Phi}_{A}\left(\tau_{k+1}, \xi\right) z(\xi) d \xi
\end{aligned}
$$

With this discrete format of governing dynamic equations the discussion on linearization is enclosed. The next section conveys how these equations fit into the successive convexification framework.

\section{Successive Convexification}

This section conveys the successive convexification approach to solve the linearized and discretized non-convex problem. The non-convex sources are convexified, after which the manner of iteratively solving the SOCP sub-problem sequence is described. For the purpose of feasibility of the initially dynamic artificial trajectory, trust regions and a virtual control parameter are introduced. The section concludes by providing the convex sub-problem and the algorithm description.

\section{A. Control Convexification}

The derivation is initialized by linearizing the lower bound constraint on the aerodynamic force and moment, as these are the only remaining nonconvex sources. For this objective two new functions $g$ and $h$ are 
defined, such that $g: \mathbb{R}^{3} \rightarrow \mathbb{R}$ and such that $h: \mathbb{R}^{3} \rightarrow \mathbb{R}:$

$$
\begin{array}{r}
g\left(\mathbf{u}_{1: 3}(\tau)\right)=F_{\mathcal{A}, \min }-\|\left.\mathbf{u}_{\mathbf{1 : 3}}(\tau)\right|_{2} \leq 0 \\
h\left(\mathbf{u}_{\mathbf{4}: \mathbf{6}}(\tau)\right)=M_{\mathcal{A}, \min }-\|\left.\mathbf{u}_{\mathbf{4 : 6}}(\tau)\right|_{2} \leq 0
\end{array}
$$

Applying a first order Taylor series to this time normalized constraint yields:

$$
\begin{array}{ccc}
F_{\mathcal{A}, \text { min }} \leq \mathbf{B}_{g}(\tau) \mathbf{u}_{\mathbf{1 : 3}}(\tau) & \text { with } & \mathbf{B}_{g}(\tau)=\frac{\hat{\mathbf{u}}_{\mathbf{1 : 3}}^{T}(\tau)}{\left\|\hat{\mathbf{u}}_{\mathbf{1 : 3}}(\tau)\right\|_{2}} \\
M_{\mathcal{A}, \min } \leq \mathbf{B}_{h}(\tau) \mathbf{u}_{\mathbf{4 : 6}}(\tau) \quad \text { with } & \mathbf{B}_{h}(\tau)=\frac{\hat{\mathbf{u}}_{\mathbf{4 : 6}}^{T}(\tau)}{\left\|\hat{\mathbf{u}}_{\mathbf{4}: \mathbf{6}}(\tau)\right\|_{2}}
\end{array}
$$

\section{B. Trust Regions and Virtual Control}

The main working principle of the successive convexification method is to solve a sequence of relaxed convex problems in an iterative manner, with $i$ denoting the $i^{\text {th }}$ iteration. For this methodology to work properly, the control regions must maintain feasible and bounded throughout the landing sequence. This entails that the cost function is to be augmented to mitigate the risk of unbound solutions. To start with, the following parameters are defined:

$$
\delta \mathbf{x}_{k}^{i}=\mathbf{x}_{k}^{i}-\mathbf{x}_{k}^{i-1}, \quad \forall k \in \mathcal{K} \delta \mathbf{u}_{k}^{i}=\mathbf{u}_{k}^{i}-\mathbf{u}_{k}^{i-1}, \quad \forall k \in \mathcal{K}
$$

Subsequently, defining $\Delta^{i} \in \mathbb{R}_{+}^{K}$, the constraint is entrenched:

$$
\delta \mathbf{x}_{k}^{i} \cdot \delta \mathbf{x}_{k}^{i}+\delta \mathbf{u}_{k}^{i} \cdot \delta \mathbf{u}_{k}^{i} \leq \mathbf{n}_{k} \cdot \Delta^{i}, \quad \forall k \in \mathcal{K}
$$

The cost function is augmented accordingly through the addition of weight term $w_{\Delta}^{i} \in \mathbb{R}_{++}$:

$$
c_{\Delta}^{i}=w_{\Delta}^{i}\left\|\Delta^{i}\right\|_{2}
$$

Artificial infeasibility is encountered when convergence is not benign. This may occur when the dynamics are linearized about a flight-time that is impractically short. This is especially true for the first few iterations when initial guesses are poor. Alleviating this problem is commonly done through the introduction of a virtual control term $\overline{\boldsymbol{v}}^{i} \in \mathbb{R}^{13(K-1)}$ with a coupled weight factor $w_{v} \in \mathbb{R}_{++}$:

$$
\begin{aligned}
\overline{\boldsymbol{v}}^{i} & =\left[\begin{array}{lll}
\overline{\boldsymbol{v}}_{0}^{i T} & \ldots & \overline{\boldsymbol{v}}_{K-2}^{i}{ }^{T}
\end{array}\right]^{T} \\
c_{v}^{i} & =w_{v}\left\|\overline{\boldsymbol{v}}^{i}\right\|_{1}
\end{aligned}
$$

In case of substantial infeasibility, the control parameter acts to prevent the algorithm from losing convergence. The weight of parameter is adjusted in accordance with the solution quality of each iteration till a certain desired threshold is achieved.

\section{Convex Sub-Problem}

In this paper the approach is taken to solve the convex sub-problems repeatedly. The goal of the discrete-time sub-problem is to minimize control, while also minimizing the trust region and virtual control. The relaxed non-convex (i.e., convex) 6DoF optimization problem is characterized in Problem 2. 


\section{Problem 2: Convex optimization problem definition in discrete time:}

Cost Function:

$$
\min _{\mathbf{u}_{k}} w_{v}\left\|\overline{\boldsymbol{v}}^{i}\right\|_{1}+w_{\Delta}^{i}\left\|\bar{\Delta}^{i}\right\|_{2} \quad \text { subject to: }
$$

Dynamics:

$$
\mathbf{x}_{k+1}^{i}=\overline{\mathbf{A}}_{k}^{i} \overline{\mathbf{x}}_{k}^{i}+\overline{\mathbf{B}}_{k}^{i} \overline{\mathbf{u}}_{k}^{i}+\overline{\mathbf{C}}_{k}^{i} \overline{\mathbf{u}}_{k+1}^{i}+\overline{\mathbf{z}}_{k}^{i}+\overline{\boldsymbol{v}}_{k}^{i}
$$

State Constraints:

$$
\begin{aligned}
\tan \gamma_{g s}\left\|H_{23}^{T} \mathbf{r}_{I, k}^{i}\right\|_{2} & \leq \mathbf{n}_{1} \cdot \mathbf{r}_{I, k}^{i} \\
\cos \theta_{\max } & \leq 1-2\left(\left(q_{k, 2}^{i}\right)^{2}+\left(q_{k, 3}^{i}\right)^{2}\right) \\
\left\|\boldsymbol{\omega}_{\mathcal{B}, k}^{i}\right\|_{2} & \leq \omega_{\max } \\
\mathbf{v}_{k}^{i} \cdot \mathbf{n}_{2} & \leq \tan \left(\alpha_{\max }\right) \mathbf{v}_{k}^{i} \cdot \mathbf{n}_{1}
\end{aligned}
$$

Control Constraints:

$$
\begin{aligned}
F_{\mathcal{A}, \min } & \leq \mathbf{B}_{g}\left(\tau_{k}\right) \mathbf{u}_{\mathbf{k}, \mathbf{1 : 3}}^{\mathbf{i}} \\
\left\|\mathbf{u}_{\mathbf{k}, \mathbf{1}: \mathbf{3}}^{\mathbf{i}}\right\|_{2} & \leq F_{\mathcal{A}, \max } \\
0 & \leq \delta_{s} \leq 1 \\
M_{\mathcal{A}, \min } & \leq \mathbf{B}_{h}\left(\tau_{k}\right) \mathbf{u}_{\mathbf{k}, \mathbf{4}: \mathbf{6}}^{\mathbf{i}} \\
\left\|\mathbf{u}_{\mathbf{k}, \mathbf{4}: \mathbf{i}}^{\mathbf{i}}\right\|_{2} & \leq M_{\mathcal{A}, \max } \\
0 & \leq \delta_{a} \leq 1
\end{aligned}
$$

Trust Regions:

$$
\delta \mathbf{x}_{k}^{i} \cdot \delta \mathbf{x}_{k}^{i}+\delta \mathbf{u}_{k}^{i} \cdot \delta \mathbf{u}_{k}^{i} \leq \Delta_{k}^{i}
$$

Boundary Conditions:

$$
\begin{aligned}
\mathbf{r}_{I, \mathbf{0}}^{\mathbf{i}} & =\mathbf{r}_{\mathcal{I}, \mathbf{i}}, \quad \mathbf{v}_{\mathcal{I}, \mathbf{0}}^{\mathbf{i}}=\mathbf{v}_{\mathcal{I}, \mathbf{i}}, \quad \boldsymbol{\omega}_{\mathcal{B}, 0}^{i}=\boldsymbol{\omega}_{\mathcal{B}, i}, \quad \mathbf{q}_{\mathcal{B} / \mathcal{I}, 0}^{i}=\left[\begin{array}{llll}
1 & 0 & 0 & 0
\end{array}\right]^{T} \\
\mathbf{r}_{\mathcal{I}, \mathbf{K}}^{\mathbf{i}} & =\mathbf{0}, \quad \mathbf{v}_{\mathcal{I}, \mathbf{L}}^{\mathbf{i}}=\mathbf{0}, \quad \boldsymbol{\omega}_{\mathcal{B}, K}^{i}=\mathbf{0}, \quad \mathbf{q}_{\mathcal{B} / \mathcal{I}, K}^{i}=\left[\begin{array}{llll}
1 & 0 & 0 & 0
\end{array}\right]^{T}
\end{aligned}
$$

\section{Successive Algorithm Description}

In this section the algorithm description for the successive convexification method is conferred. To start with, tolerances on the trust region and virtual control are defined as $\Delta_{\text {tol }} \in \mathbb{R}_{++}$and $v_{\text {tol }} \in \mathbb{R}_{++}$, respectively. The algorithm is initialized and run in a successive manner. The loop is run until the solution is within the desired threshold tolerance values, or the maximum number of iterations $i_{\max }$ is reached. 


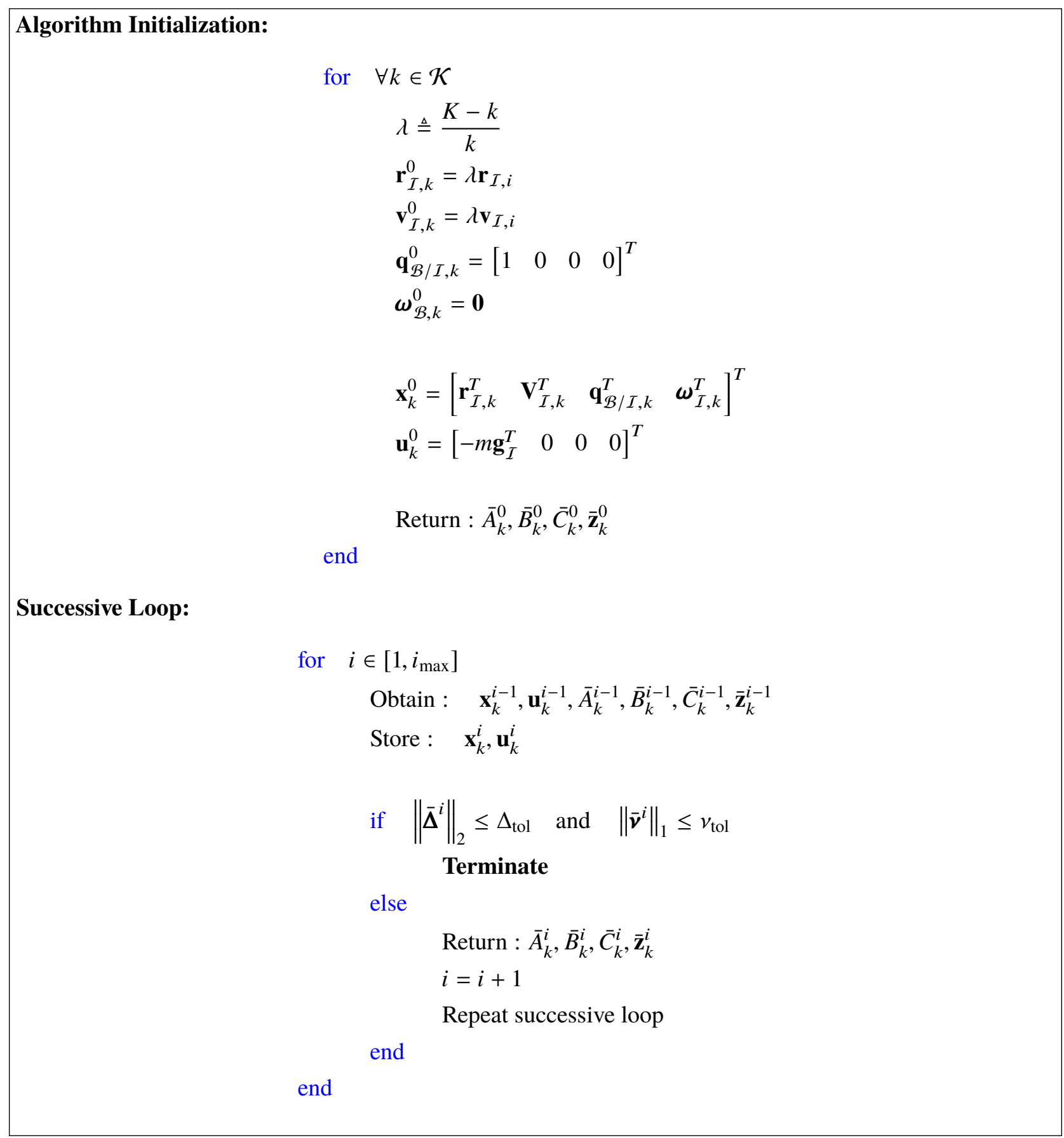

\section{Simulations}

The simulation results for the designed Titan parafoil descent algorithm are presented in this section. All simulations were generated using MATLAB, specifically CVX [13] and SDPT3 [14]. The simulation parameters, algorithm parameters and starting conditions are provided in Table 3 . These starting conditions are in-line with prior work on Titan precision landing prior to terminal descent.

From Fig. 5 it can be observed that the position and velocity terminate at zero magnitude, while the soft-landing algorithm is successfully adhering to imposed state and control constraints. Both the control authorities, i.e., symmetric and asymmetric deflections, remain within the desired normalized values zero and 
Table 3 Simulation parameters, algorithm parameters and starting conditions.

* Updated parafoil surface area value based on internal JPL discussions.

\begin{tabular}{lll}
\hline \hline Simulation Parameters & Description & Value \\
\hline$g_{T}$ & Titan gravity & $1.352 \mathrm{~m} / \mathrm{s}^{2}$ \\
$m$ & Spacecraft mass & $201.4 \mathrm{~kg}$ \\
$S_{r e f} *$ & Parafoil surface area & $7.25 \mathrm{~m}^{2}$ \\
$t_{f}$ & Time-of-flight & $76 \mathrm{~s}$ \\
$\alpha_{\max }$ & Maximum angle-of-attack & $45^{\circ}$ \\
$\gamma_{g s}$ & Glide-slope angle & $20^{\circ}$ \\
$\theta_{\max }$ & Maximum tilt angle & $90^{\circ}$ \\
$\omega_{\max }$ & Maximum angular rate & $30^{\circ} / \mathrm{s}$ \\
\hline Algorithm Parameters & Description & Value \\
\hline$w_{v}$ & Virtual control weight factor & $10^{5}$ \\
$w_{\Delta}^{i}$ & Trust region weight factor & $10^{-3}$ \\
$v_{\text {tol }}$ & Virtual control tolerance & $10^{-9}$ \\
$\Delta_{\text {tol }}$ & Trust region tolerance & $10^{-3}$ \\
$i_{\max }$ & Maximum number of iterations & 10 \\
$K$ & Temporal nodes & 70 \\
\hline Starting Conditions & Description & Value \\
\hline $\mathbf{r}_{\mathbf{0}}$ & Starting position & {$[9,-7,183]^{T} \mathrm{~m}$} \\
$\mathbf{v}_{\mathbf{0}}$ & Starting velocity & {$[9,0,-8]^{T} \mathrm{~m} / \mathrm{s}$} \\
\hline \hline
\end{tabular}

one, as becomes evident from Figs. 6a and 6b, respectively. Interesting remark is that the control authorities seem to take turns in controlling the parafoil to the target, with an overlap period of several seconds. From the attitude rate plot (Fig. 7) it becomes evident that control authority in east-direction is rather limited. This is in accordance with the established equations, as the deflections have no control over the side-force nor the pitching moment. This issue, as depicted in Fig. 7a, could be tackled through inclusion of a propulsion system. The quaternion attitude representation is restricted to have the unit quaternion equal one, which is the case as deduced from Fig. 7b The trajectories in Fig. 8 visualize the motion both in-plane and out-of-plane. From the 2D-trajectory, up-east specifically, it becomes evident that at termination, the spacecraft is turned back in opposite direction of the wind, which is an imposed constraint on the system. As shown in the 3D plot, the simulation has been run for various initial state conditions to assess the algorithm's robustness.

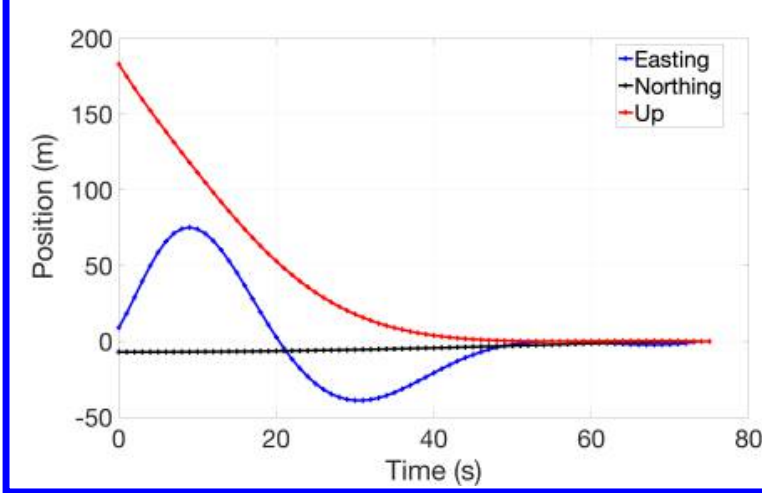

(a) Position profile

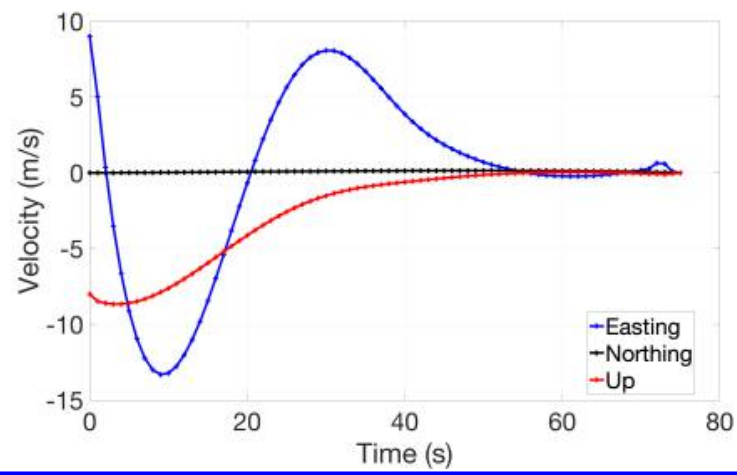

(b) Velocity profile

Fig. 5 Time history of the position and velocity states. 


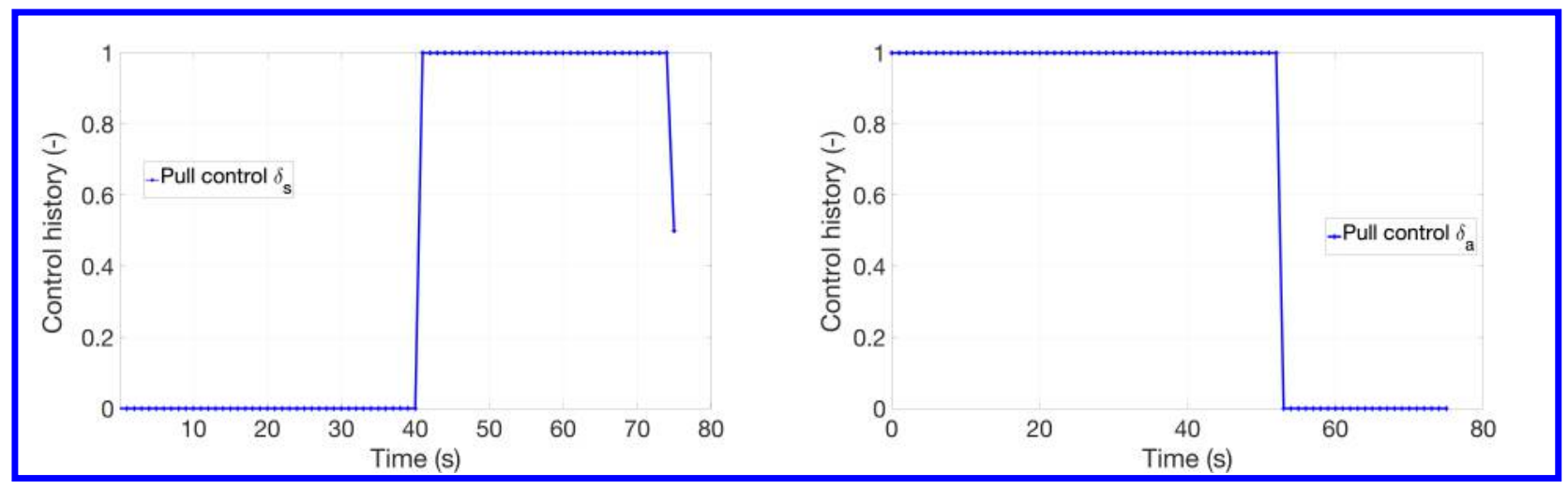

(a) Symmetric control profile

(b) Asymmetric control profile

Fig. 6 Time history of the control deflections.

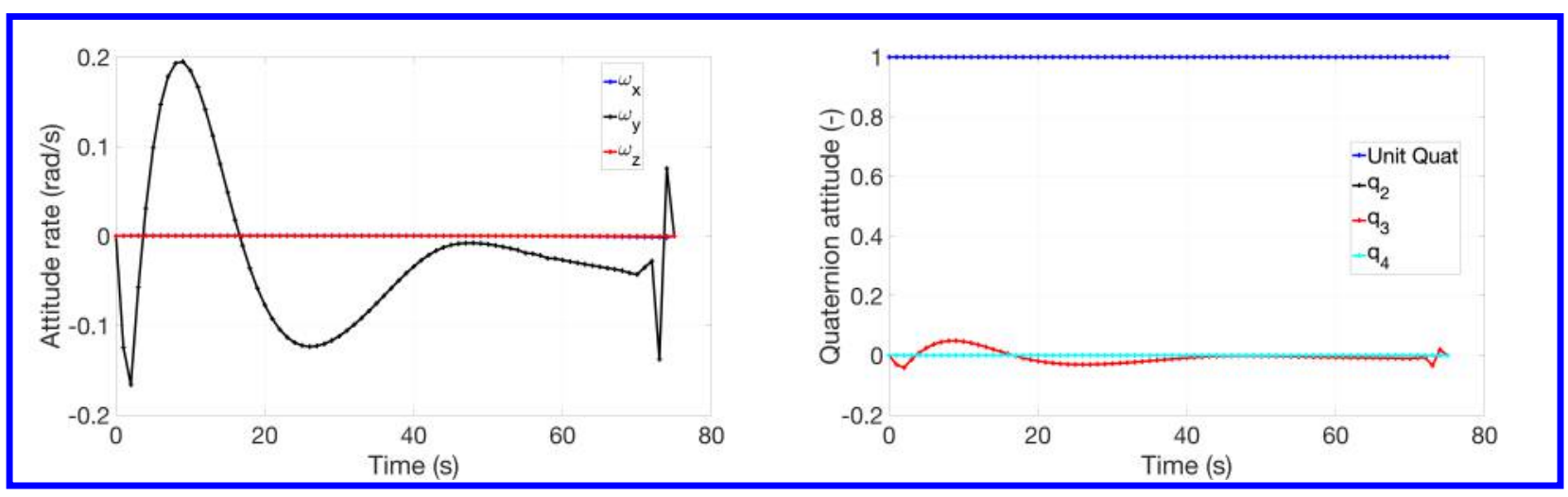

(a) Attitude rate profile

(b) Quaternion attitude profile

Fig. 7 Time history of angular rates and quaternion angles.

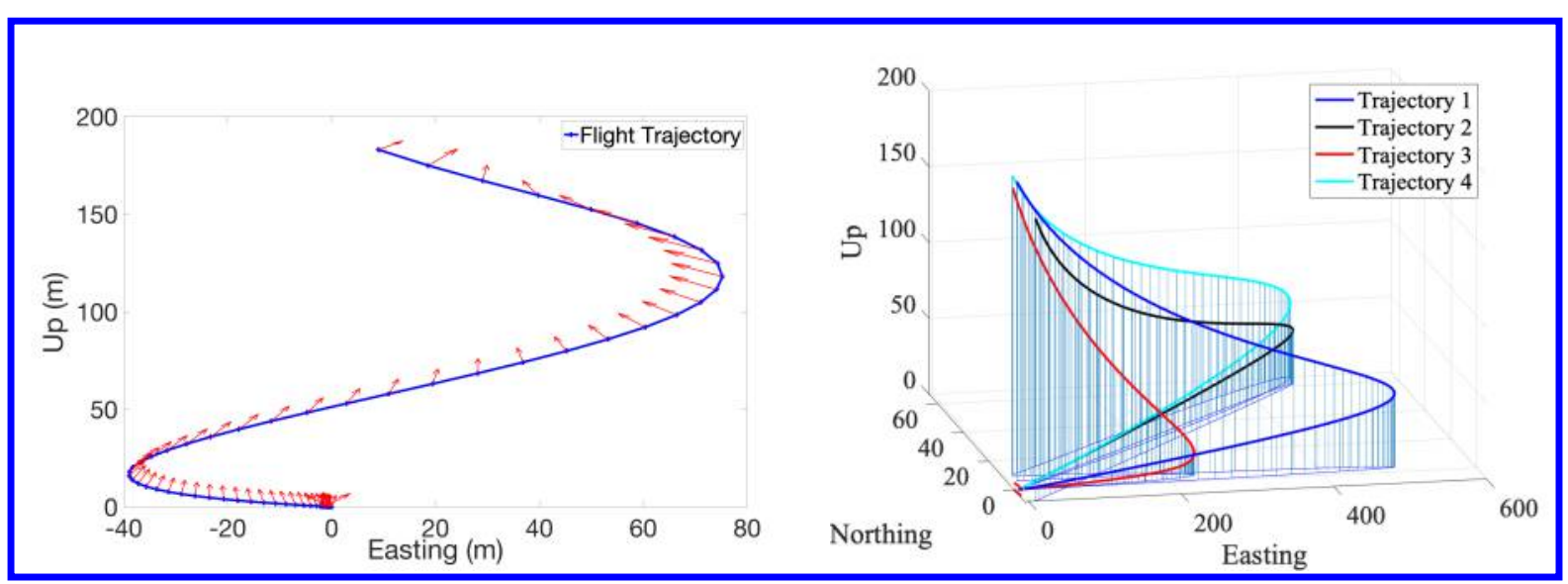

(a) Trajectory visualization (in-plane)

(b) Trajectories visualization (out-of-plane)

Fig. 8 In-plane and out-of-plane path representations. 
This section ends with a sensitivity analysis, which provides great insight into how initial state conditions may effect the flight trajectory, the control effort and the attitude. Through this process the robustness of the parafoil guidance algorithm is typically evaluated. Based on the simulation results it is even possible to point out possible risk factors that influence the landing precision or the flight trajectory in general. The parameters that are included in the sensitivity study have been captivated in Table 4 Notice that these values have been altered for a total of six cases: adding 5\%,10\% and 20\% to, and reducing 5\%, 10\% and 20\% from the nominal initial state conditions. While the percentages are relative, absolute values have been used in the simulations. The results are now conferred for each scenario, presenting time histories of the position, velocity, throttle setting, the trajectory, and the attitude dynamics and kinematics. These entities have been chosen as they provide the most valuable simulation information.

Table 4 Parameters of sensitivity and their respective percentages.

\begin{tabular}{lll}
\hline \hline Parameter & Description & Sensitivity interval \\
\hline $\mathbf{r}_{0}$ & Initial position & $\pm 20 \%$ \\
$\mathbf{v}_{0}$ & Initial velocity & $\pm 20 \%$ \\
\hline \hline
\end{tabular}

In Figs. 9-14 the sensitivity simulation results are presented. To maintain a clear discussion, a short discussion and some conclusions per varied variable are conferred:

- Initial position sensitivity: presented in Figs. 9-11. From the position profile the conclusion is drawn that despite the imposed variation, the algorithm behaves in a similar fashion. On the other hand, the total control history varies extremely with variation in initial position. While the control still adheres to the imposed bound between 0 and 1, the fluctuations are large. The switch logic might get too excessive for on-board control. The algorithm no longer follows a distinct max-min-max profile. The quaternion and attitude profiles show stable variations just like the position and velocity profiles.

- Initial velocity sensitivity: presented in Figs. 12-14. The observations are very much similar to the previous case. Additionally, it is denoted that the control fluctuations are to some extent extremer. This is expected, as the velocity directly governs the aerodynamics.

The conclusion is drawn that the algorithm is very well capable of guiding the parafoil to the target for a wide range of starting state conditions in terms of position and velocity. For actual flight implementation a minimum error guidance algorithm is required to relax the optimal control problem.

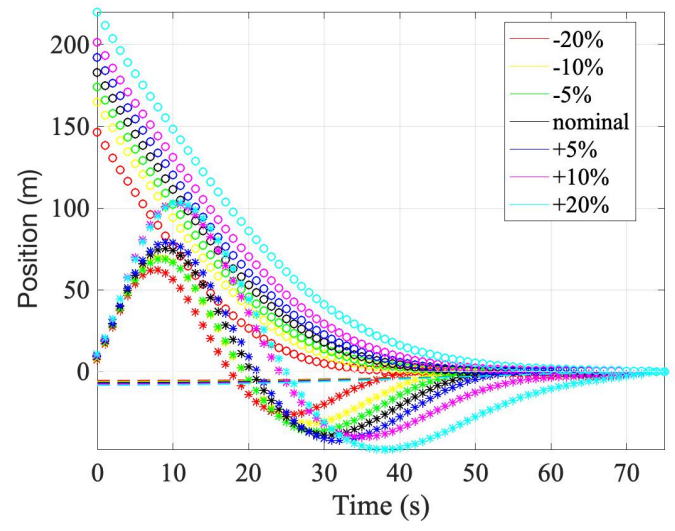

(a) Position profile

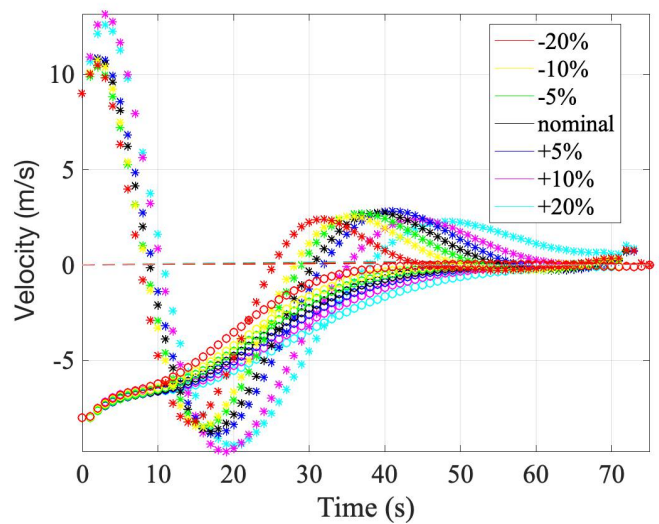

(b) Velocity profile

Fig. 9 Position and velocity time histories due to initial position sensitivity of $\pm 20 \%$. 


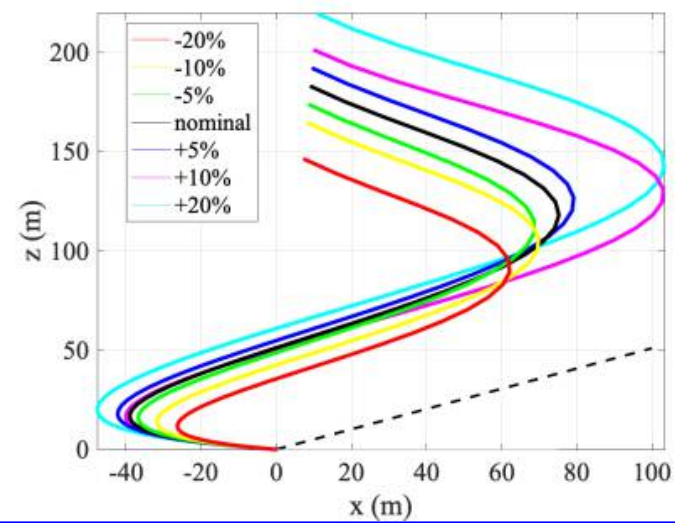

(a) Trajectory

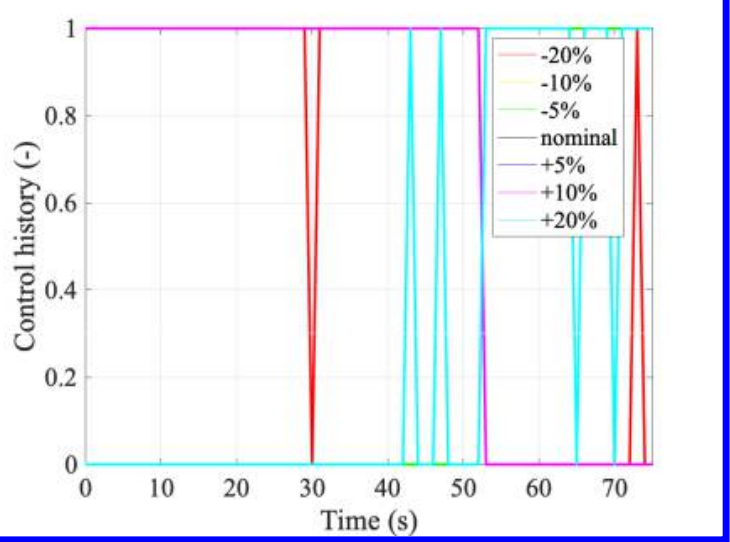

(b) Control profile

Fig. 10 Trajectory and total control history due to initial position sensitivity of $\pm 20 \%$.

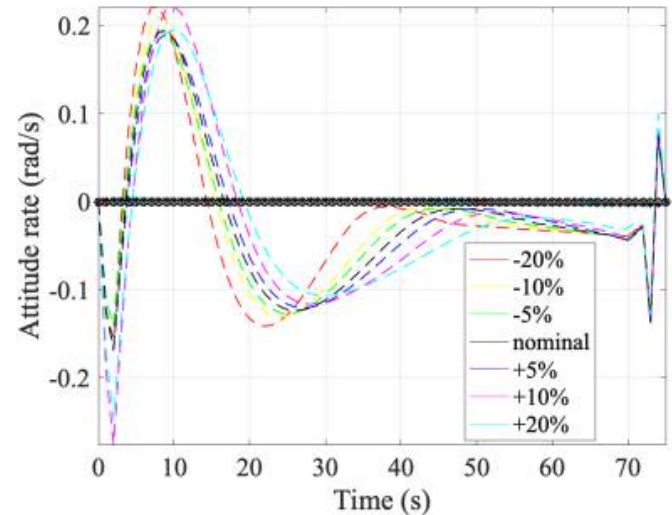

(a) Attitude rate profile

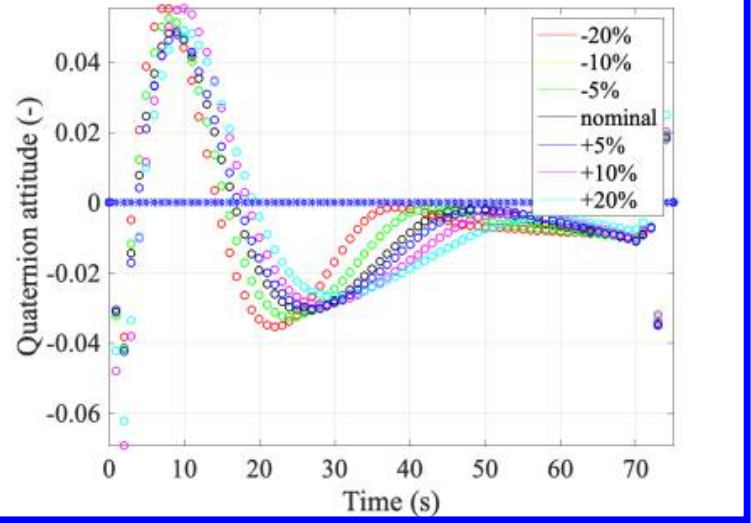

(b) Quaternion attitude profile

Fig. 11 Attitude and quaternion attitude time histories due to initial position sensitivity of $\pm 20 \%$.

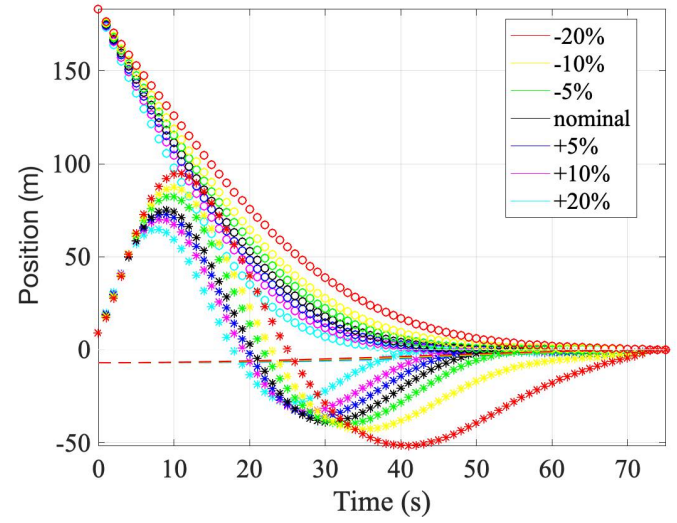

(a) Position profile

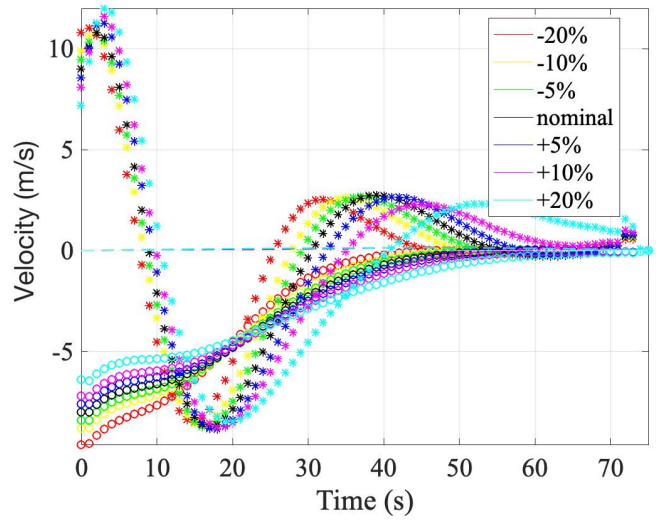

(b) Velocity profile

Fig. 12 Position and velocity time histories due to initial velocity sensitivity of $\pm 20 \%$. 


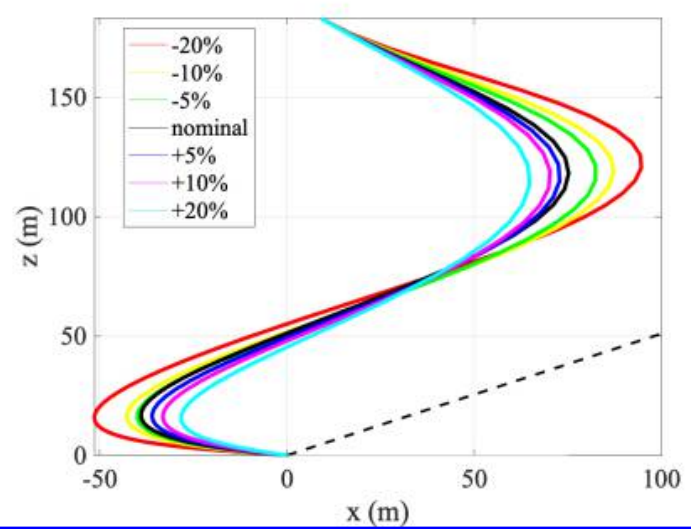

(a) Trajectory

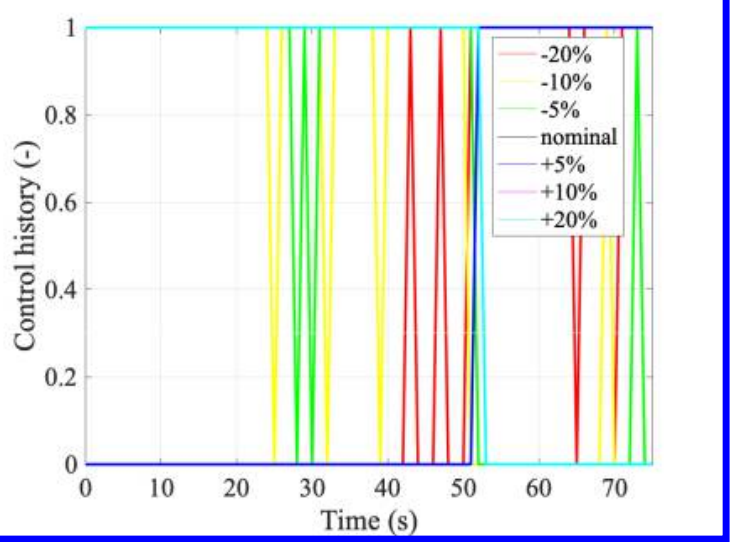

(b) Control profile

Fig. 13 Trajectory and total control history due to initial velocity sensitivity of $\pm 20 \%$.

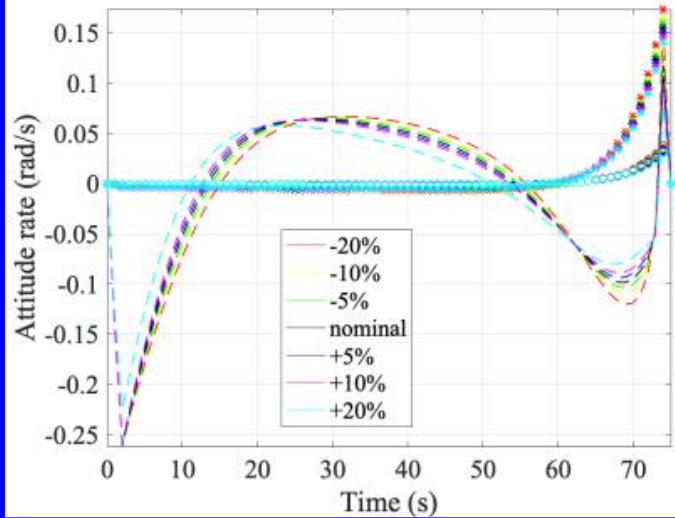

(a) Attitude rate profile

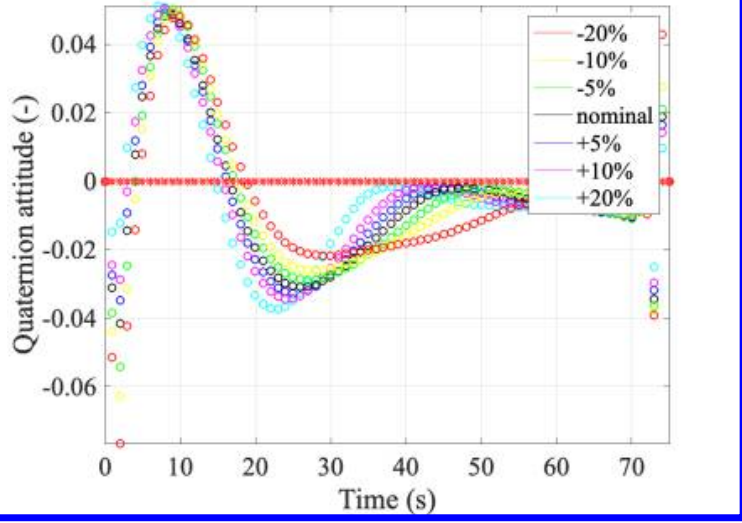

(b) Quaternion attitude profile

Fig. 14 Attitude and quaternion attitude time histories due to initial velocity sensitivity of $\pm 20 \%$.

\section{Conclusions and Recommendations}

This paper has adapted the successive convex optimization method for simulating Titan parafoil terminal descent sequences, with a distinct focus on guidance formulated as a finite horizon optimal control problem. All simulations have been carried using CVX in Matlab. The soft-landing is guaranteed while adhering to imposed mission constraints. The control time history remains within desired limits. The glide-slope constraint has been properly enforced to ensure the spacecraft does not reach the surface fast, which would cause an undesired sub-surface flight. Likewise, the pointing constraint has been enforced successfully to cope with the requirements of TRN. The conclusion is drawn that the algorithm is very well capable of guiding the parafoil to the target for a wide range of starting state conditions in terms of position and velocity.

The algorithm is to be extended as a 9DoF high-fidelity model within NASA/JPL sophisticated Dynamics And Real-Time Simulation (DARTS) tool, to test for real-time hardware design and visualize terminal descent. Testing in $\mathrm{C}++$ is advised to guarantee fast convergence. Including thrusters and on-board fuel (to a certain allowable mass) is also advised for Titan terminal descent to increase control authority, especially for the motion in and around the easting direction. Future work also includes the implementation of more inclusive wind models, as this heavily influences the motion of a parafoil in Titan's dense atmosphere. 


\section{Acknowledgments}

(C2020 California Institute of Technology. Government sponsorship acknowledged. This research was carried out at the Jet Propulsion Laboratory, California Institute of Technology, under a contract with the National Aeronautics and Space Administration. This research was carried out in collaboration with the Astrodynamics and Space Missions Department of Delft University of Technology, The Netherlands.

\section{References}

-11] Quadrelli, M. B., Schutte, A., Rimani, J., and Ermolli, L., "Aero Maneuvering Dynamics and Control for Precision Landing on Titan,” 2019 IEEE Aerospace Conference, Big Sky, MT, USA, 2019, pp. 1-16. doi: 10.1109/AERO.2019.8742230.

-[2] Hörst, S. M., "Titan's Atmosphere and Climate," Journal of Geophysical Research: Planets, Vol. 122, No. 3, 2017, pp. 432-482. doi:0.1002/2016JE005240.

[3] Lunine, J. I., "Saturn's Titan: A strict test for life's cosmic ubiquity," Proceedings of the American Philosophical Society, Vol. 153, 2009, pp. 404-419.

-[4] Mooij, E., "Characteristic Motion of Re-entry Vehicles," AIAA Atmospheric Flight Mechanics (AFM) Conference, Guidance, Navigation, and Control and Co-located Conferences, American Institute of Aeronautics and Astronautics, 2013. doi:10.2514/6.2013-4603.

[5] Mazouz, R., "Autonomous Guidance and Control for Precision Landing on Planetary Bodies: Convex Optimization Approach For Mars and Titan Case Studies,” Delft University of Technology, 2019.

-[6] Yakimenko, O., Precision Aerial Delivery Systems: Modeling, Dynamics, and Control, Vol. 248, Progress in Astronautics and Aeronautics, AIAA, 2015.

[7] Szmuk, M., and Acikmese, B., "Successive Convexification for 6-DoF Mars Rocket Powered Landing with Free-Final-Time," 2018 AIAA Guidance, Navigation, and Control Conference, Kissimmee, FL, 2018. doi: $10.2514 / 6.2018-0617$.

[8] Szmuk, M., Acikmese, B., and Berning, A. W., "Successive Convexification for Fuel-Optimal Powered Landing with Aerodynamic Drag and Non-Convex Constraints," AIAA Guidance, Navigation, and Control Conference, San Diego, CA, 2016. doi:10.2514/6.2016-0378.

-[9] Markley, F. L., "Attitude Error Representations for Kalman Filtering," Journal of Guidance, Control, and Dynamics, Vol. 26, No. 2, 2003, pp. 311-317.

-[10] Lissaman, P., and Brown, G., "Apparent mass effects on parafoil dynamics," Aerospace Design Conference, Meeting Paper Archive, American Institute of Aeronautics and Astronautics, 1993. doi:10.2514/6.1993-1236.

[11] Yelle, R., Strobel, D., Lellouch, E., and Gautier, D., "Engineering Models for Titan’s Atmosphere,” Tech. rep., 1998.

[12] Kowaleczko, G., "Apparent masses and inertia moments of the parafoil," Journal of Theoretical and Applied Mechanics, Vol. 52, No. 3, 2014, pp. 606-616.

-[13] Liu, X., and Lu, P., "Solving Nonconvex Optimal Control Problems by Convex Optimization," Journal of Guidance, Control, and Dynamics, Vol. 37, No. 3, 2014, pp. 750-765.

-[14] Toh, K., and Todd, M., "SDPT3 - a MATLAB software package for semidefinite programming," Optimization Methods and Software, Vol. 11, No. 1, 1999, pp. 545-581. 